\title{
A Nonlinear Planar Beam Formulation with Stretch and Shear Deformations Under End Forces and Moments
}

\author{
H. Ren \\ MSC Software Corporation \\ 201 Depot Street \\ Ann Arbor, MI 48105, USA \\ Email: hui.ren@mscsoftware.com \\ Telephone: 734-994-3800 ext. 4071
}

\author{
W. D. Zhu* \\ Department of Mechanical Engineering \\ University of Maryland, Baltimore County \\ 1000 Hilltop Circle \\ Baltimore, MD 21250, USA \\ Email:wzhu@umbc.edu \\ Telephone: 410-455-3394
}

\author{
W. Fan \\ Department of Mechanical Engineering \\ University of Maryland, Baltimore County \\ 1000 Hilltop Circle \\ Baltimore, MD 21250, USA \\ Email: fanwei@umbc.edu
}

\begin{abstract}
A new nonlinear planar beam formulation with stretch and shear deformations is developed in this work to study equilibria of a beam under arbitrary end forces and moments. The slope angle and stretch strain of the centroid line, and shear strain of cross-sections, are chosen as dependent variables in this formulation, and end forces and moments can be either prescribed or resultant forces and moments due to constraints. Static equations of equilibria are derived from the principle of virtual work, which consist of one second-order ordinary differential equation and two algebraic equations. These equations are discretized using the finite difference method, and equilibria of the beam can be accurately calculated. For practical, geometrically nonlinear beam problems, stretch and shear strains are usually small, and a good approximate solution of the equations can be derived from the solution of the corresponding Euler-Bernoulli beam problem. The bending deformation of the beam is the only important one in a slender beam, and stretch and shear strains can be derived it, which give a theoretical validation of the accuracy and applicability of the nonlinear Euler-Bernoulli beam formulation. Relations between end forces and moments and relative displacements of two ends of the beam can be easily calculated. This formulation is powerful in the study of buckling of beams with various boundary conditions under compression, and can be used to calculate post-buckling equilibria of beams. Higher-order buckling modes of a long slender beam that have complex configurations are also studied using this formulation.
\end{abstract}

\section{INTRODUCTION}

Developing geometrically nonlinear models for beams and cables is an important subject in academic research $[1,2]$ and engineering applications such as compliant mechanisms [3-5] and micro- and nano-electro-mechanical systems [6,7]. In compliant mechanisms, a pseudo-rigid-body (PRB) model for a beam is usually developed for their design and analysis. An analytical solution for the beam under end loads would be useful. Elliptic-integral solutions for a large-deflection EulerBernoulli cantilever beam with end loads have been given in Ref. [3]. While they are valid only for simple geometries and loadings, they have been widely used in developing various PRB models $[4,5]$.

\footnotetext{
${ }^{*}$ Corresponding author.
} 
Formulations of beams and cables are generally considered to be different, but in practice, it is difficult to make such a distinction. Irvine [8] pointed out that a long slender beam behaves like a cable in the large; on the other hand, the small bending stiffness of a slack cable can be important in calculation of its equilibrium and dynamic response [9]. The study of equilibria of long slender beams is usually referred to as Kirchhoff-Love rod theory [1], and many researchers have made contributions to this area. Santillan et al. [10] theoretically and experimentally studied equilibria and stability of an elastic beam with two ends clamped together. Antman [2] provided a detailed derivation of equations of a beam using the rod theory, and Svetlitsky [11] discussed a similar topic from an engineering viewpoint. Kim and Chirikjian [12] studied equilibria and free vibration characteristics of a rod using a group-theoretical approach. Hodges [13] provided a systematic study on formulations of beams made of composite materials with arbitrary cross-sections, which can describe both slender and thick beams. Kumar [14] developed a generic model for partial delamination in composite beams using the finite element method (FEM). Romero et al. [15] developed a torsion-free non-linear beam model for beam-like slender structures whose cross sections can withstand only traction, shear, and bending.

In geometrically exact beam theories [16-18], spatial discretization of shear strains of cross sections can lead to some numerical issue called shear locking. Pai [19] pointed out that shear locking can be caused by combining the bending rotation and the shear rotation into one bending-shear rotation variable and reducing the order of the beam theory such as the Timoshenko beam theory. In numerical studies of nonlinear beams such as the FEM [20] and the absolute nodal coordinate formulation (ANCF) [21], bending and stretch deformations can be easily considered, but the shear deformation is usually neglected since it is so small, which leads to an Euler-Bernoulli beam model [22]. A new planar Rayleigh beam model was developed by Zhu et al. [9] using the slope angle of its centroid line. It uses much fewer number of generalized coordinates compared with the FEM. In practice, the magnitude of the shear strain can be larger than that of the stretch strain; a nonlinear model with stretch but without shear would not be appropriate in this case.

In this work, a planar beam is described by the slope angle and stretch strain of its centroid line, and shear strains of cross sections, so that bending, stretch, and shear deformations can be fully described, which extends the work in [9] where only the slope angle is considered. Static equations of equilibria are derived using the principle of virtual work and discretized using the finite difference method (FDM). The shear strain is expressed as a dependent variable, and the discretized model has no shear locking. When stretch and shear strains are small, an approximate solution can be obtained from a solution of the corresponding Euler-Bernoulli beam problem. Approximate stretch and shear strain distributions along the beam are determined from the slope angle distribution and compared with those from the FDM. Buckling loads of beams [23] are studied using the current formulation, and post-buckling equilibria of beams with various boundary conditions are accurately calculated. Higher-order buckling modes of a long slender beam that have complex configurations are also studied using the current formulation.

\section{Nonlinear Formulation of a Planar Beam Under End Forces and Moments}

Consider a planar beam of length $L$ shown in Fig. 1. A cross-section of the beam can be described by an undeformed arc-length coordinate $s$ along its centroid line, where $0 \leq s \leq L$. Suppose that cross-sections of the beam remain to be planar after deformation. General deformation of a planar beam includes bending and stretch deformations of its centroid line,
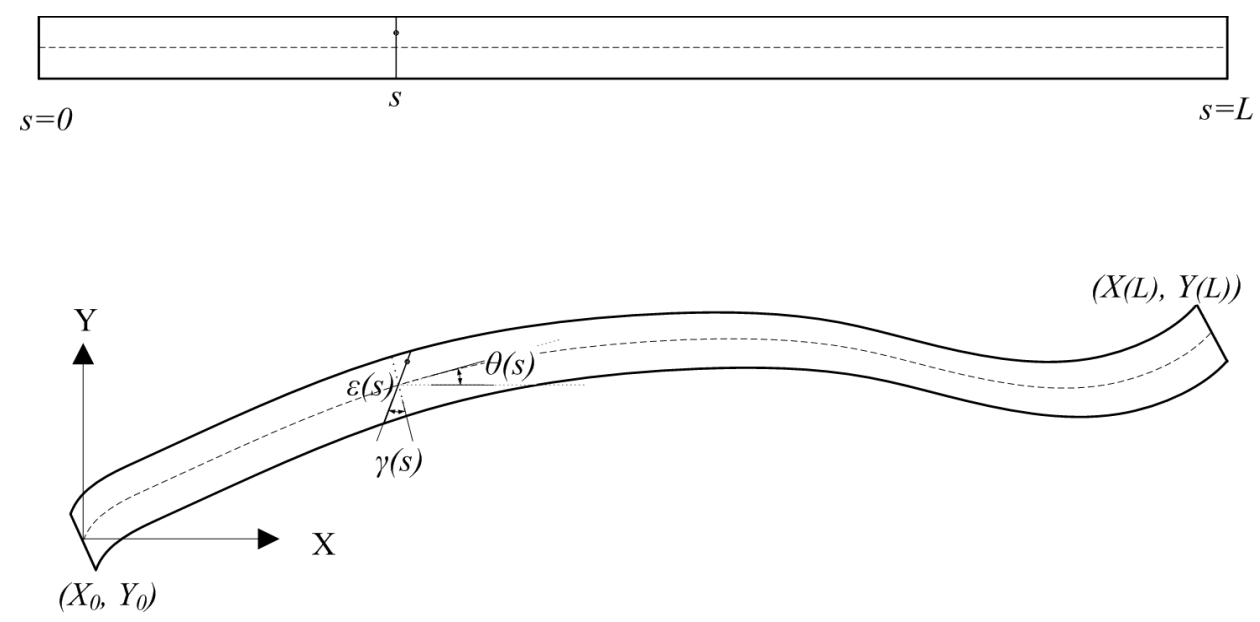

Fig. 1. Description of the configuration of a planar beam with large deformation using its slope angle $\theta(s)$, stretch strain $\varepsilon(s)$, and shear angle or strain $\gamma(s)$. 
and shear angles or strains of its cross-sections. The slope angle $\theta(s)$, the shear strain $\gamma(s)$, and the stretch strain $\varepsilon(s)$ are used to describe the configuration of the deformed beam, and spatial coordinates of the particle on the centroid line at the cross-section corresponding to the arc-length coordinate $s$ are

$$
X(s)=X_{0}+\int_{0}^{s} \cos \theta(\tau)[1+\varepsilon(\tau)] d \tau, \quad Y(s)=Y_{0}+\int_{0}^{s} \sin \theta(\tau)[1+\varepsilon(\tau)] d \tau
$$

where $\left(X_{0}, Y_{0}\right)$ are coordinates of the particle on the centroid line at the cross-section corresponding to the arc-length coordinate $s=0$. The elastic potential energy of the beam is

$$
\mathcal{P}=\frac{1}{2} \int_{0}^{L}\left\{E A(s) \varepsilon^{2}(s)+E I(s)\left[\frac{d \theta(s)}{d s}-\frac{d \gamma(s)}{d s}\right]^{2}+\frac{G A(s)}{k(s)} \gamma^{2}(s)\right\} d s,
$$

where $E$ is the Young's modulus, $G$ is the shear modulus, $k(s)$ is the shear correction factor of the cross-section corresponding to the arc-length coordinate $s$, and $A(s)$ and $I(s)$ are the area and moment of inertia of the cross-section; the derivation is shown in Appendix A. Let

$$
\phi(s)=\theta(s)-\gamma(s)
$$

which is the bending angle of the cross-section corresponding to the arc-length coordinate $s$. The elastic potential energy in Eq. (2) becomes

$$
\mathcal{P}=\frac{1}{2} \int_{0}^{L}\left[E A(s) \varepsilon^{2}(s)+E I(s)\left(\frac{d \phi(s)}{d s}\right)^{2}+\frac{G A(s)}{k(s)} \gamma^{2}(s)\right] d s,
$$

and its variation is

$$
\delta P=\left.E I(s) \phi^{\prime}(s) \delta \phi(s)\right|_{0} ^{L}-\int_{0}^{L}\left(E I(s) \phi^{\prime}(s)\right)^{\prime} \delta \phi(s) d s+\int_{0}^{L} E A(s) \varepsilon(s) \delta \varepsilon(s) d s+\int_{0}^{L} \frac{G A(s)}{k(s)} \gamma(s) \delta \gamma(s) d s,
$$

where a prime denotes differentiation with respect to $s$. Suppose that there are external concentrated forces $\mathbf{F}_{0}=\left(F_{x}^{0}, F_{y}^{0}\right)^{T}$ and $\mathbf{F}_{1}=\left(F_{x}^{1}, F_{y}^{1}\right)^{T}$, and external concentrated moments $m_{0}$ and $m_{1}$ applied at two ends of the beam, and these forces and moments can be either prescribed or resultant forces and moments due to constraints. By Eqs. (1) and (3), one has

$$
X(L)=X_{0}+\int_{0}^{L}\{\cos [\phi(s)+\gamma(s)][1+\varepsilon(s)]\} d s, \quad Y(L)=Y_{0}+\int_{0}^{L}\{\sin [\phi(s)+\gamma(s)][1+\varepsilon(s)]\} d s .
$$

The virtual work done by the external forces and moments are

$$
\delta \mathcal{W}=F_{x}^{0} \delta X_{0}+F_{y}^{0} \delta Y_{0}+m_{0} \delta \phi(0)+F_{x}^{1} \delta X(L)+F_{y}^{1} \delta Y(L)+m_{1} \delta \phi(L)
$$

Substituting Eq. (6) into Eq. (7) yields

$$
\begin{gathered}
\delta \mathcal{W}=\left(F_{x}^{0}+F_{x}^{1}\right) \delta X_{0}+\left(F_{y}^{0}+F_{y}^{1}\right) \delta Y_{0}+m_{0} \delta \phi(0)+m_{1} \delta \phi(L)+\int_{0}^{L}\left[F_{y}^{1} \cos (\phi+\gamma)-F_{x}^{1} \sin (\phi+\gamma)\right](1+\varepsilon) \delta \phi d s \\
+\int_{0}^{L}\left[F_{y}^{1} \cos (\phi+\gamma)-F_{x}^{1} \sin (\phi+\gamma)\right](1+\varepsilon) \delta \gamma d s+\int_{0}^{L}\left[F_{x}^{1} \cos (\phi+\gamma)+F_{y}^{1} \sin (\phi+\gamma)\right] \delta \varepsilon d s .
\end{gathered}
$$

Force and moment balance equations and static equations of equilibria of the beam are derived from the principle of virtual work $\delta P=\delta \mathcal{W}$ :

$$
\begin{gathered}
F_{x}^{0}=-F_{x}^{1}, \quad F_{y}^{0}=-F_{y}^{1}, \quad-E I(0) \phi^{\prime}(0)=m_{0}, \quad E I(L) \phi^{\prime}(L)=m_{1}, \\
-\left(E I(s) \phi^{\prime}(s)\right)^{\prime}=\left\{F_{y}^{1} \cos [\phi(s)+\gamma(s)]-F_{x}^{1} \sin [\phi(s)+\gamma(s)]\right\}[1+\varepsilon(s)]=\frac{G A(s)}{k(s)} \gamma(s), \\
E A(s) \varepsilon(s)=F_{x}^{1} \cos [\phi(s)+\gamma(s)]+F_{y}^{1} \sin [\phi(s)+\gamma(s)],
\end{gathered}
$$


where the last two equations are static equations of equilibria. If both $F_{x}^{1}$ and $F_{y}^{1}$ are prescribed, without loss of generality, suppose

$$
F_{x}^{1}=-F \cos \bar{\theta}, \quad F_{y}^{1}=-F \sin \bar{\theta}
$$

and Eq. (9) becomes

$$
-\left(E I(s) \phi^{\prime}(s)\right)^{\prime}=F \sin [\phi(s)+\gamma(s)-\bar{\theta}][1+\varepsilon(s)]=\frac{G A(s)}{k(s)} \gamma(s), \quad E A(s) \varepsilon(s)=-F \cos [\phi(s)+\gamma(s)-\bar{\theta}] .
$$

\section{Study of a Uniform Beam}

For a uniform beam, $A$ and $I$ are constant alone the beam. Define dimensionless constants

$$
\delta=\frac{F}{E A}, \quad \alpha=\frac{F L^{2}}{E I}, \quad \varsigma=\frac{I}{A L^{2}}=\frac{\delta}{\alpha}, \quad \sigma=\frac{E}{G},
$$

and dimensionless variables

$$
\xi=\frac{s}{L}, \quad x=\frac{X}{L}, \quad y=\frac{Y}{L}, \quad \psi(\xi)=\phi(s), \quad \imath(\xi)=\gamma(s), \quad \varepsilon(\xi)=\varepsilon(s), \quad \vartheta(\xi)=\theta(s) .
$$

Equation (11) becomes

$$
-\psi^{\prime \prime}(\xi)=\alpha \sin [\psi(\xi)+\mathfrak{l}(\xi)-\bar{\theta}][1+\varepsilon(\xi)], \quad \mathfrak{\imath}(\xi)=k \varpi \delta \sin [\psi(\xi)+\mathfrak{l}(\xi)-\bar{\theta}][1+\varepsilon(\xi)], \quad \varepsilon(\xi)=-\delta \cos [\psi(\xi)+\mathfrak{l}(\xi)-\bar{\theta}] .
$$

In the study of mechanics of materials [24], a beam is considered to be slender or thick depending on the ratio of its length over its height. If the ratio is greater than 10 , the beam is considered to be slender; otherwise, it is considered to be thick. It is easy to calculate that when the ratio of the length over the height of a beam is $10, \varsigma=\frac{1}{1200}$. Hence, in general, $\varsigma$ can be considered as the slenderness parameter of a beam. If $\varsigma<10^{-3}$, the beam is considered to be slender, and if $\varsigma>10^{-3}$, it is considered to be thick. Moreover, if the beam is made of an isotropic material, $\Phi=2(1+v)$, where $v$ is Poisson's ratio, and if it is made of a composite material, $\varpi$ needs to be specified.

In practice, stretch and shear strains in a beam are usually small. By Eq. (14), the stretch strain is in the order of $\delta$, and the shear strain is in the order of $k \varpi \delta$, which indicate that $\delta=\alpha \varsigma \ll 1$ and $k \varpi \delta \ll 1$. Hence, solutions of Eq. (14) can be expanded by series of $\delta$ :

$$
\psi(\xi)=\bar{\psi}(\xi)+\mathrm{O}(\delta), \quad \varepsilon(\xi)=\mathrm{O}(\delta), \quad \mathfrak{\imath}(\xi)=\mathrm{O}(\delta)
$$

where $\bar{\psi}(\xi)$ satisfies

$$
-\bar{\psi}^{\prime \prime}(\xi)=\alpha \sin [\bar{\psi}(\xi)-\bar{\theta}]
$$

and first-order terms of $\varepsilon$ and $\imath$ are

$$
\varepsilon(\xi)=-\delta \cos [\bar{\psi}(\xi)-\bar{\theta}]+\mathrm{O}\left(\delta^{2}\right), \quad \mathfrak{\imath}(\xi)=k \varpi \delta \sin [\bar{\psi}(\xi)-\bar{\theta}]+\mathrm{O}\left(\delta^{2}\right)
$$

respectively. The function $\bar{\psi}(\xi)$ can be calculated from Eq. (16), and $\varepsilon(\xi)$ and $\imath(\xi)$ can be subsequently calculated from Eq. (17). Relative errors of the above approximations are in order of $\delta$, which is usually accurate enough for engineering applications, especially for long slender beams. This explains why the nonlinear Euler-Bernoulli beam formulation can usually give accurate results. Moreover, stretch and shear strains are usually of the same order, and the shear strain could be even larger than the stretch strain, in which case an accurate beam model should include both strains at the same time. In the FEM and the ANCF, the shear strain is sometimes neglected to avoid shear locking, but the stretch strain is considered, which 
may not be appropriate in some cases. Multiplying both sides of Eq. (16) by $\bar{\psi}^{\prime}(\xi)$ and integrating the resulting expression with respect to $\xi$ yield

$$
\frac{1}{2}\left(\frac{d \bar{\psi}(\xi)}{d \xi}\right)^{2}-\alpha \cos [\bar{\psi}(\xi)-\bar{\theta}]=\text { const }
$$

where the integration constant can be determined using boundary conditions of the beam. When $\delta \ll 1$, a good approximation of $\psi$ can be obtained from Eq. (18). Using the result in Eq. (17) yields good approximations of $\varepsilon$ and $\gamma$.

\section{Cantilever Beams}

For a uniform cantilever beam, without loss of generality, suppose

$$
X_{0}=0=Y_{0}, \quad \psi(0)=0
$$

and the slope angle at the free end of the beam satisfies

$$
\psi^{\prime}(1)=\frac{m_{1} L}{E I} \equiv \bar{\kappa} .
$$

Equations (14), (19), and (20) can be discretized as follows:

1. Partition the unit length of the beam into $\mathrm{N}$ elements using nodes $\xi_{n}=\frac{n}{N}$, where $n=0,1, \cdots, N$, with the element length $h=\frac{1}{N}$.

2. Discretize the first two equations in Eq. (14) using central difference to yield

$$
\begin{gathered}
N^{2} \psi_{n-1}-2 N^{2} \psi_{n}+\alpha \sin \left(\psi_{n}+\mathfrak{l}_{n}-\bar{\theta}\right)\left[1-\delta \cos \left(\psi_{n}+\mathfrak{l}_{n}-\bar{\theta}\right)\right]+N^{2} \psi_{n+1}=0, \quad n=1,2, \cdots, N-1, \\
\mathfrak{l}_{n}-k \bar{\omega} \delta \sin \left(\psi_{n}+\mathfrak{l}_{n}-\bar{\theta}\right)\left[1-\delta \cos \left(\psi_{n}+\mathfrak{l}_{n}-\bar{\theta}\right)\right]=0, \quad n=0,1, \cdots, N,
\end{gathered}
$$

which are $2 N$ algebraic equations with $2 N+2$ unknowns $\psi_{n}$ and $\varepsilon_{n}$, where $n=0,1, \cdots, N$.

3. The two boundary conditions, which are the second equation in Eq. (19) and Eq. (20), give two more equations:

$$
\psi_{0}=0, \quad N^{2} \psi_{N-2}-4 N^{2} \psi_{N-1}+3 N^{2} \psi_{N}=2 \bar{\kappa}
$$

4. Values of $\psi_{n}$ and $\mathfrak{l}_{n}$, where $n=0,1, \cdots, N$, can be calculated from Eqs. (21) and (22). Stretch strains at the nodes are

$$
\varepsilon_{n}=-\delta \cos \left(\psi_{n}+\mathfrak{l}_{n}-\bar{\theta}\right), \quad n=0,1, \cdots, N
$$

5. The equilibrium of the beam can be calculated from Eq. (1):

$$
x_{n}=\int_{0}^{\xi_{n}} \cos (\psi+\mathfrak{\imath})(1+\varepsilon) d \eta, \quad y_{n}=\int_{0}^{\xi_{n}} \sin (\psi+\mathfrak{l})(1+\varepsilon) d \eta
$$

Boundary conditions for Eq. (16) are

$$
\bar{\psi}(0)=0, \quad \bar{\psi}^{\prime}(1)=\bar{\kappa} .
$$

Substituting the second equation in Eq. (25) into Eq. (18) yields

$$
\frac{1}{2}\left(\frac{d \bar{\psi}(\xi)}{d \xi}\right)^{2}-\alpha \cos [\bar{\psi}(\xi)-\bar{\theta}]=\frac{\bar{\kappa}^{2}}{2}-\alpha \cos [\bar{\psi}(1)-\bar{\theta}]
$$

Equation (26) can usually be easily solved. The solution of Eq. (26) and the corresponding approximate solution in Eq. (17) can be used as a good initial guess to iteratively solve Eqs. (21) and (22). 


\subsection{A Uniform Cantilever Beam with a Concentrated Moment at Its Free End}

As shown in Fig. 2, only a moment $m$ is applied at the free end of a cantilever beam, and one has $\alpha=\delta=0$ by Eq. (12). Hence,

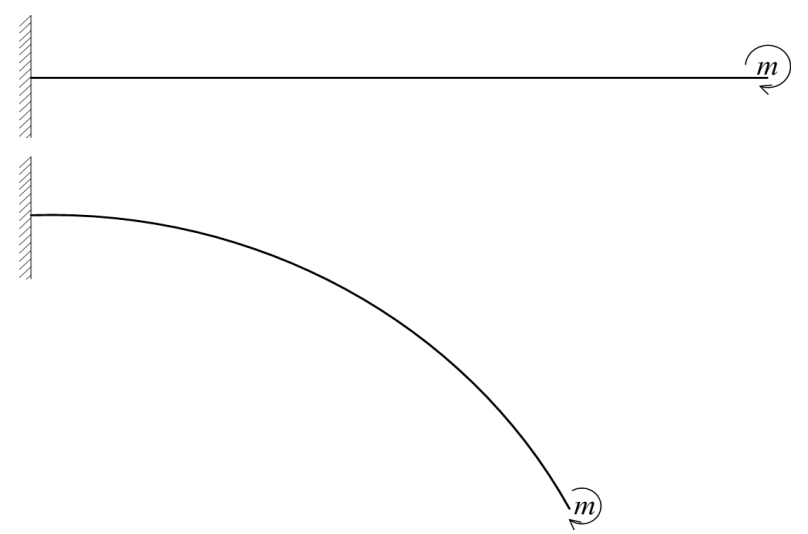

Fig. 2. A cantilever beam with a concentrated moment at its free end.

$$
\varepsilon(\xi)=0, \quad \imath(\xi)=0, \quad \bar{\kappa}=\frac{m L}{E I}, \quad \psi(\xi)=\vartheta(\xi)
$$

There is neither stretch nor shear deformation in the beam, and cross sections of the beam are always perpendicular to its centroid line. Equation (26) becomes

$$
\frac{d \psi(\xi)}{d \xi}=\bar{\kappa}
$$

which means that the curvature of the beam is constant along the beam. By the second equation in Eq. (19), one has

$$
\psi(\xi)=\vartheta(\xi)=\bar{\kappa} \xi, \quad \varepsilon(\xi)=\imath(\xi)=0
$$

Then the equilibrium of the beam can be calculated as

$$
X(s)=\frac{E I}{m} \sin \frac{m s}{E I}, \quad Y(s)=\frac{E I}{m}\left(1-\cos \frac{m s}{E I}\right),
$$

which is an arc with a constant curvature $\frac{m}{E I}$.

A similar result can be obtained for a non-uniform beam. Equation (11) becomes

$$
-\left(E I(s) \phi^{\prime}(s)\right)^{\prime}=0, \quad \frac{G A(s)}{k} \gamma(s)=0, \quad E A(s) \varepsilon(s)=0
$$

with boundary conditions

$$
\phi(0)=0, \quad E I(L) \phi^{\prime}(L)=m
$$

Solving Eqs. (31) and (32) yields

$$
\phi(s)=\theta(s)=m \int_{0}^{s} \frac{d \tau}{E I(\tau)}, \quad \varepsilon(s)=0, \quad \gamma(s)=0
$$

and there is neither stretch nor shear deformation in the beam. 


\subsection{A Uniform Cantilever Beam with a Concentrated Vertical Force at Its Free End}

As shown in Fig. 3, a concentrated vertical force $F$ is applied at the free end of a cantilever beam, and one has

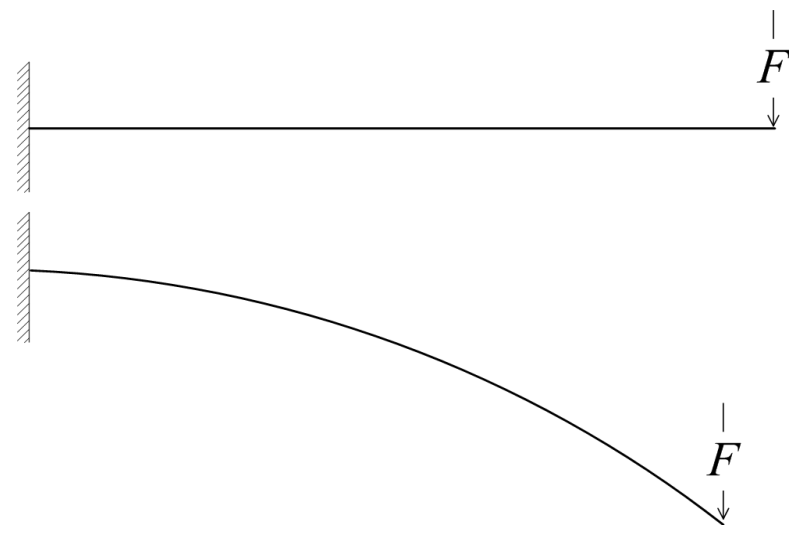

Fig. 3. A cantilever beam with a concentrated vertical force at its free end.

$$
\bar{\theta}=\frac{\pi}{2}, \quad \bar{\kappa}=0
$$

Then Eq. (26) becomes

$$
\left(\frac{d \bar{\psi}(\xi)}{d \xi}\right)^{2}=2 \alpha[\sin \bar{\psi}(\xi)-\sin \bar{\psi}(1)]
$$

which indicates that $0 \geq \bar{\psi}(\xi) \geq \bar{\psi}(1)$, and one has

$$
\int_{0}^{|\bar{\psi}(\xi)|} \frac{d \vartheta}{\sqrt{\sin |\bar{\psi}(1)|-\sin \vartheta}}=\sqrt{2 \alpha \xi}, \quad \int_{0}^{|\bar{\psi}(1)|} \frac{d \vartheta}{\sqrt{\sin |\bar{\psi}(1)|-\sin \vartheta}}=\sqrt{2 \alpha}
$$

The relation between $|\bar{\psi}(1)|$ and $\alpha$ is shown in Fig. 4, and the vertical force required to bend the beam, so that the slope angle at the free end of the beam is a prescribed angle $\bar{\psi}(1)$, is $F=\frac{\alpha E I}{L^{2}}$. Subsequently, $\bar{\psi}(\xi)$ can be calculated from the first

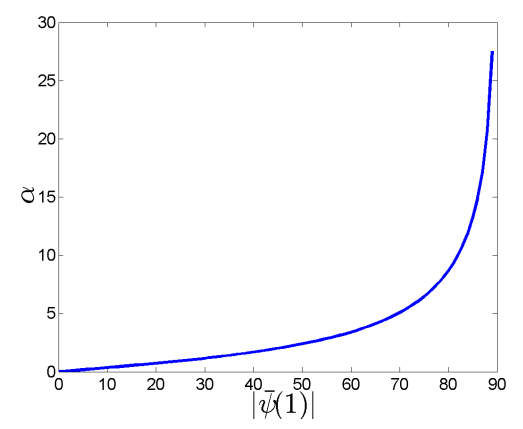

Fig. 4. The dimensionless transverse force $\alpha=\frac{F L^{2}}{E I}$ required to bend the beam so that the slope angle at the end of the beam $|\bar{\psi}(1)|$ is as prescribed (in degrees). 
equation in Eq. (36), and the corresponding $\varepsilon$ and $\imath$ can be calculated from Eq. (17). When $|\bar{\psi}(1)|=\frac{\pi}{2}$,

$$
\int_{0}^{\frac{\pi}{2}} \frac{d \vartheta}{\sqrt{\sin \frac{\pi}{2}-\sin \vartheta}}>\int_{\frac{\pi}{2}-\varepsilon}^{\frac{\pi}{2}} \frac{d \vartheta}{\sqrt{\sin \frac{\pi}{2}-\sin \vartheta}}=\int_{0}^{\varepsilon} \frac{d \varphi}{\sqrt{1-\cos \varphi}}=\sqrt{2} \int_{0}^{\frac{\varepsilon}{2}} \frac{d \frac{\varphi}{2}}{\sin \frac{\varphi}{2}}=\sqrt{2} \int_{0}^{\frac{\varepsilon}{2}} \frac{d x}{x} \frac{1}{1-\frac{x^{2}}{3 !}+\cdots}=+\infty
$$

Hence, $\alpha=\infty$. This indicates that it is impossible to bend an Euler-Bernoulli cantilever beam by only a vertical force at its free end so that the slope angle at the free end is $\frac{\pi}{2}$.

Distributions of the slope angle and stretch and shear strains, as well as equilibria of a beam, with the slenderness parameter $\varsigma=10^{-4}$, and the slope angle at the free end prescribed by $\bar{\psi}(1)=-10^{\circ},-45^{\circ},-80^{\circ}$, are shown in Fig. 5 , which correspond to $\alpha=0.3530,2.0145,8.6788$, respectively. The approximate solutions are in perfect agreement with solutions calculated by the FDM for the slender beam, but they do not agree so well for a thick beam with $\varsigma=10^{-2}$, as shown in Fig. 6, since the nonlinear Euler-Bernoulli beam formulation is accurate only for a slender beam. In Fig. 5, the magnitude of the slope angle increases along the beam, while the curvature decreases, with the maximum bending moment at the fixed end. The stretch strain is zero at the fixed end, and becomes the largest at the free end. The maximum shear strain occurs at the fixed end. Note that while magnitudes of $\psi, \varepsilon$, and $\imath$ are quite different, their profiles are similar for different vertical forces at the free end.

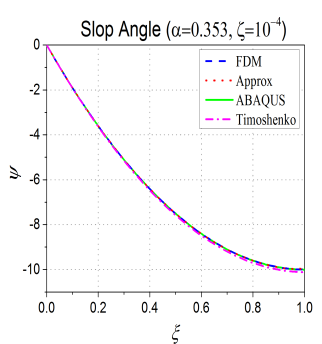

(a)

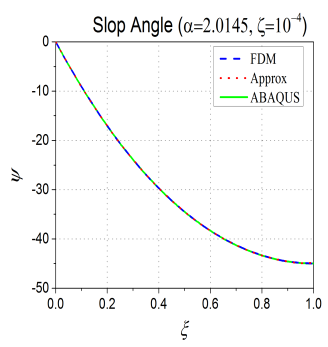

(e)

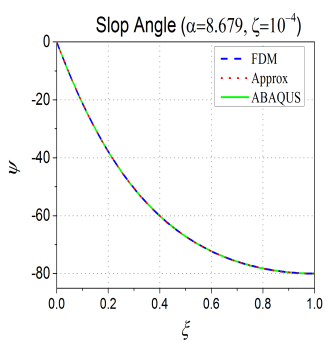

(i)

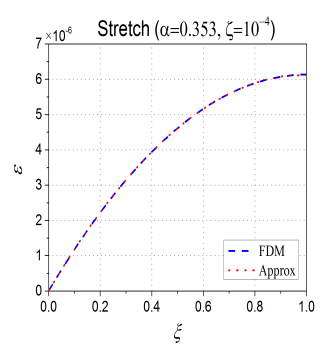

(b)

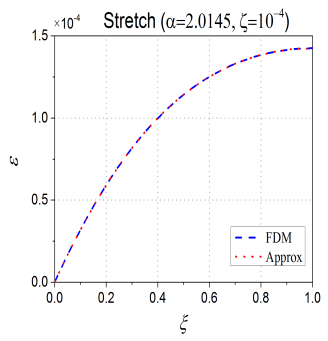

(f)

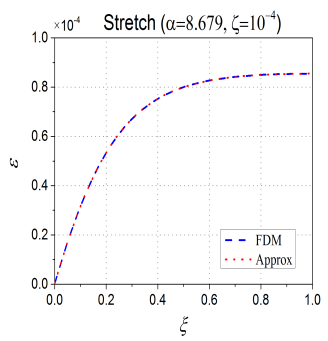

(j)

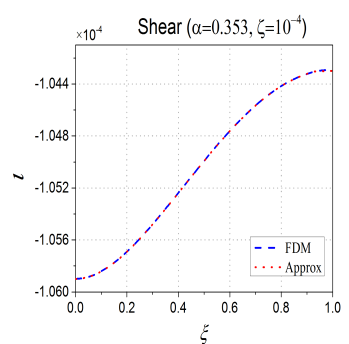

(c)

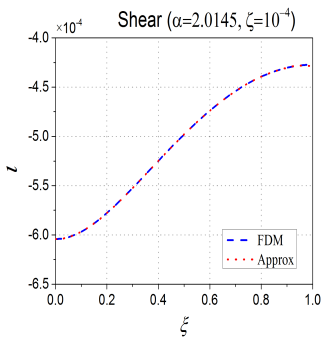

(g)

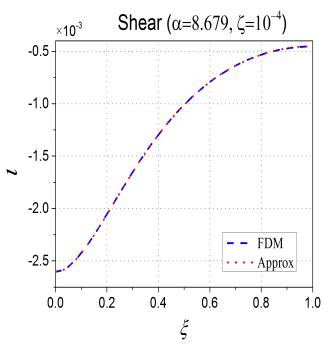

(k)

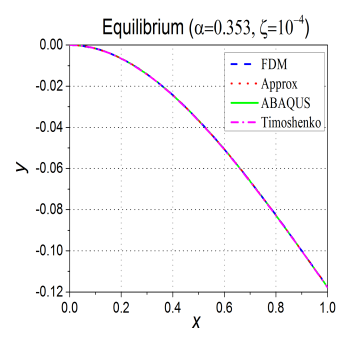

(d)

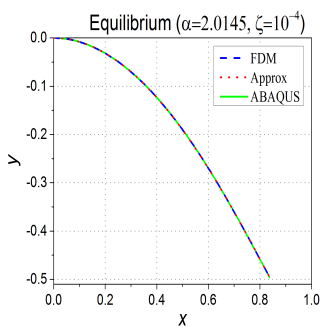

(h)

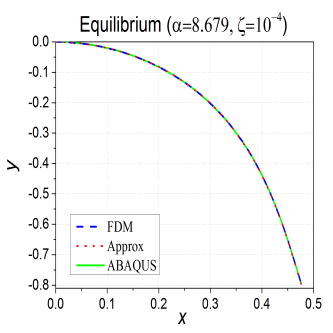

(1)

Fig. 5. Distributions of $\psi, \varepsilon$, and $\mathrm{l}$, and equilibria of a slender cantilever beam under a vertical end force, where the slope angle at the free end is: (a) through (d), $-10^{\circ}$; (e) through (h), $-45^{\circ}$; and (i) through (l), $-80^{\circ}$.

Slope angles and equilibria from the current formulation are compared with those from the Timoshenko beam theory using the FEM. Since the Timoshenko beam theory is suitable only for beams with small deformations and does not include stretch deformation, only cases with small vertical forces, which correspond to $\alpha=0.3530$, are calculated, as shown in Figs. 5(a) and 5(d) and Figs. 6(a) and 6(d). Results from the current formulation and the Timoshenko beam theory are in 


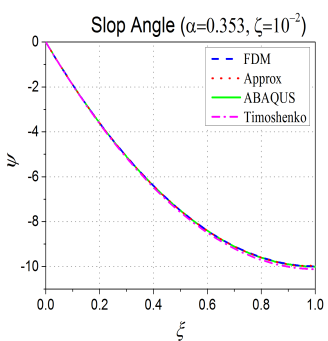

(a)

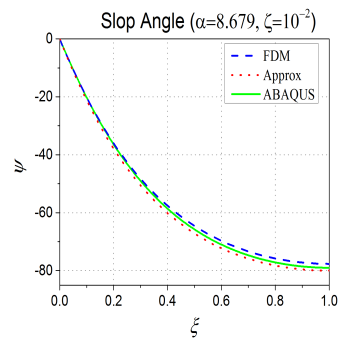

(e)

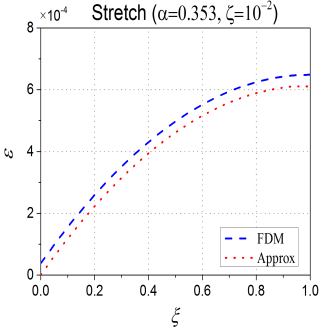

(b)

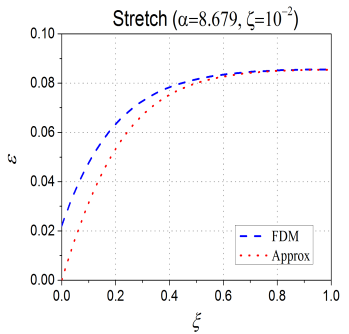

(f)

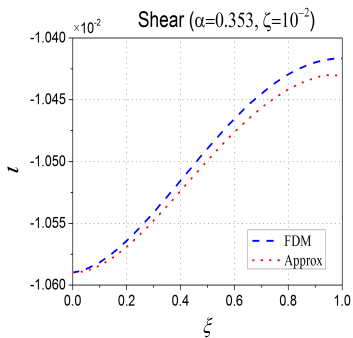

(c)

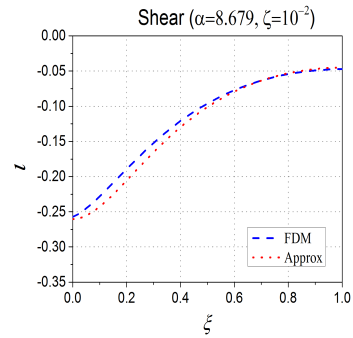

$(\mathrm{g})$

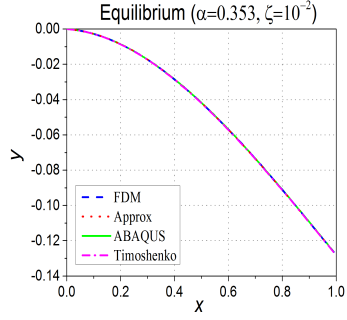

(d)

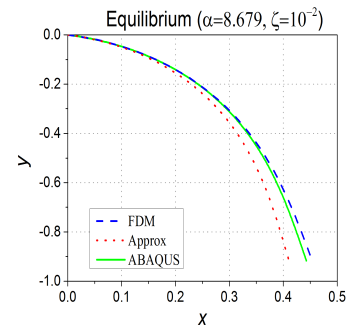

(h)

Fig. 6. Distributions of $\psi, \varepsilon$, and $\imath$, and equilibria of a thick cantilever beam under a vertical end force, when the slope angle at the free end is: (a) through (d), $-10^{\circ}$; and (e) through (h), $-80^{\circ}$.

Table 1. Deflections at the tip of the cantilever beam from different formulations

\begin{tabular}{|c|c|c|c|c|}
\hline \multirow{2}{*}{} & \multicolumn{2}{|c|}{ Timoshenko beam theory } & \multirow{2}{*}{ ABAQUS } & Current formulation \\
\cline { 2 - 5 } & Lower-order elements & High-order elements & & \\
\hline 1 element & $-4.22 \times 10^{-4}$ & -0.118 & -0.109 & - \\
\hline 2 elements & $-1.67 \times 10^{-3}$ & -0.118 & -0.114 & -0.109 \\
\hline
\end{tabular}

perfect agreement. Note that thel Timoshenko beam theory would suffer from shear locking because it does not distinguish the bending rotation and the shear rotation. If interpolation functions in the FEM are not properly chosen, the shear deformation would lock the bending deformation. When low-order elements with linear interpolation functions are used in the Timoshenko beam theory, it can show a significant phenomenon of shear locking when only one element is used, as shown in Table 1. Using high-order consistent interpolation functions for bending and shear angles would usually avoid shear locking. In the Timoshenko beam theory, the shear angle $\gamma$ is related to the lateral deflection $v$ and the bending angle $\phi$ by $\gamma=\frac{d v}{d x}-\phi$, which means interpolation functions for $\phi$ should have the same order as those for $\frac{d v}{d x}$. When high-order elements are used in the Timoshenko beam theory, where third- and second-order polynomial functions are used to interpolate the deflection $v$ and the bendingl angle $\phi$, respectively, shear locking can be avoided, as shown in Table 1.

Slope angles and equilibria from the current formulation are also compared with those from planar Timoshenko beam elements in ABAQUS in Figs. 5 and 6, and they are in excellent agreement with each other. Since three nodes are needed to discretize the second-order derivative term in the first equation in Eq. (14) using central difference in the FDM, as shown in Eq. (21), there is no solution from the current formulation when only one element is used, as shown in Table 1 . Unlike the Timoshenko beam theory, the current formulation and ABAQUS can avoid shear locking and deal with large deformation of a beam; the current formulation can also accurately calculate the stretch strain, as shown in Fig. 5. While the transverse shear stiffness is accounted for in planar Timoshenko beam elements in ABAQUS, the shear strain is not available in its output for users. When the magnitude of the shear strain is larger than that of the stretch strain, the stretch strain would not be accurately calculated from ABAQUS.

\subsection{A Uniform Cantilever Beam with a Concentrated Transverse Force at Its Free End}

Consider the equilibrium of a cantilever beam, where a concentrated transverse force $F$ is applied at its free end, which is always perpendicular to the tangent of the centroid line of the beam at the free end, as shown in Fig. 7. Components of the force are $\mathbf{F}=(F \sin \theta(L),-F \cos \theta(L))^{T}$, and one has 


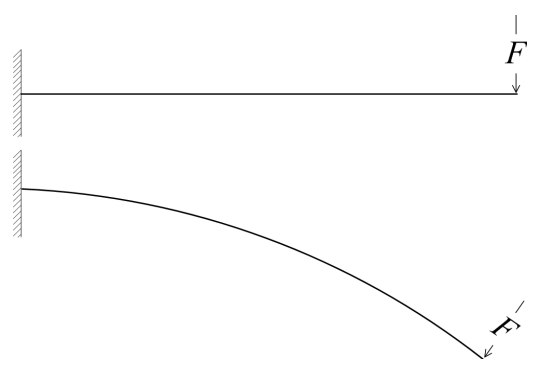

Fig. 7. A cantilever beam with a concentrated transverse force at its free end.

$$
\bar{\theta}=\frac{\pi}{2}+\bar{\psi}(1), \quad \bar{\kappa}=0
$$

Equation (16) becomes

$$
\left(\frac{d \bar{\psi}(\xi)}{d \xi}\right)^{2}=2 \alpha \sin [\bar{\psi}(\xi)-\bar{\psi}(1)]
$$

which indicates that $0 \geq \bar{\psi}(\xi) \geq \bar{\psi}(1)$, and one has

$$
\int_{\bar{\psi}(\xi)}^{0} \frac{d \vartheta}{\sqrt{\sin [\vartheta-\bar{\psi}(1)]}}=\sqrt{2 \alpha} \xi, \quad \int_{\bar{\psi}(1)}^{0} \frac{d \vartheta}{\sqrt{\sin [\vartheta-\bar{\psi}(1)]}}=\sqrt{2 \alpha}
$$

The relation between $\bar{\psi}(1)$ and $\alpha$ is shown in Fig. 8. It is interesting to notice that the relation between the transverse force and

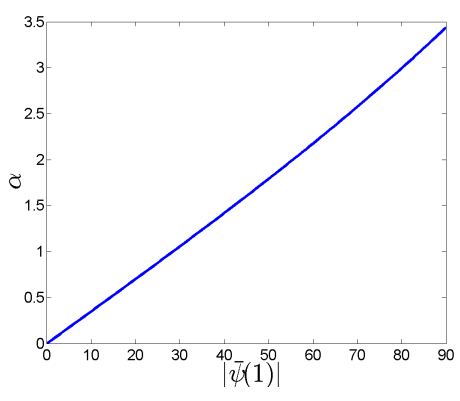

(a)

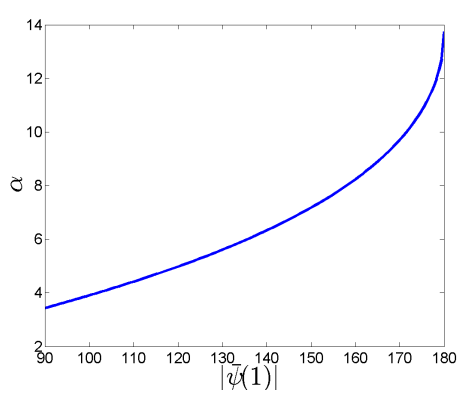

(b)

Fig. 8. Dimensionless transverse forces $\alpha$ corresponding to different slope angles $\bar{\psi}(1)$ at the free end of the beam under a transverse force there for: (a) $0 \leq|\bar{\psi}(1)| \leq \frac{\pi}{2}$, and (b) $\frac{\pi}{2} \leq|\bar{\psi}(1)| \leq \pi$.

slope angle at the free end is almost linear when $-\pi / 2 \leq \bar{\psi}(1) \leq 0$ (Fig. 8(a)), and the cantilever beam behaves like a linear spring, with the slope angle at the free end proportional to the transverse force. Distributions of $\psi, \varepsilon$, and $\imath$ along the beam, as well as equilibria of the beam with the slenderness parameter $\varsigma=10^{-4}$ are shown in Fig. 9, with the slope angle at the free end $\psi(1)=-10^{\circ},-45^{\circ},-90^{\circ},-135^{\circ},-180^{\circ}$, and correspondingly, $\alpha=0.34941,1.60413,3.4376,5.9614,13.7504$, respectively. One can see that profiles of the slope angle and stretch and shear strains are quite similar when $0 \leq|\psi(1)| \leq 90^{\circ}$; the maximum bending moment and shear strain occur at the fixed end, and the maximum stretch strain occurs at the free end. When $|\bar{\psi}(1)|>\frac{\pi}{2}$, the maximum stretch strain moves towards the interior of the beam, and the maximum bending moment and shear strain can occur at either end of the beam.

\subsection{Buckling of a Uniform Cantilever Beam Under an Axial Compressive Force}

Consider a cantilever beam under an axial compressive force $F$ applied at the free end $s=L$. One has

$$
\bar{\theta}=0, \quad \bar{\kappa}=0,
$$




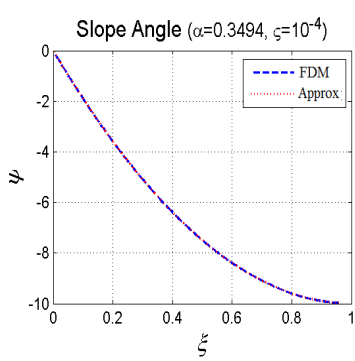

(a)

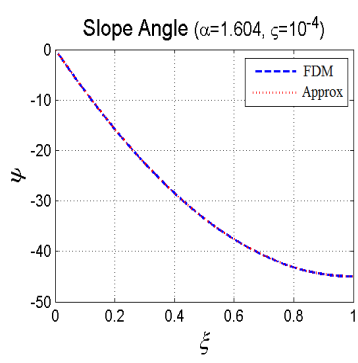

(e)

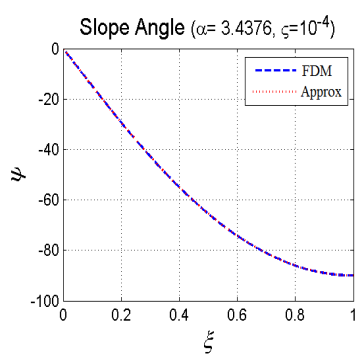

(i)

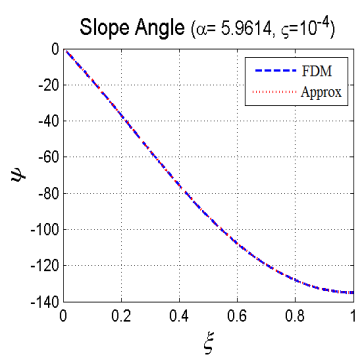

(m)

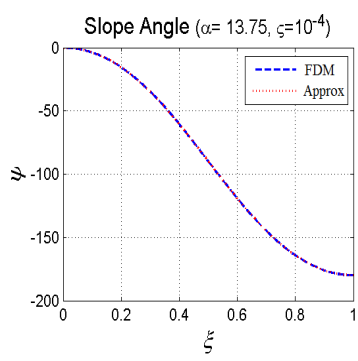

(q)

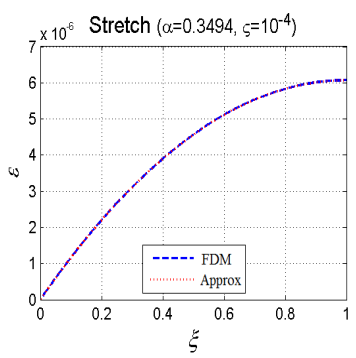

(b)

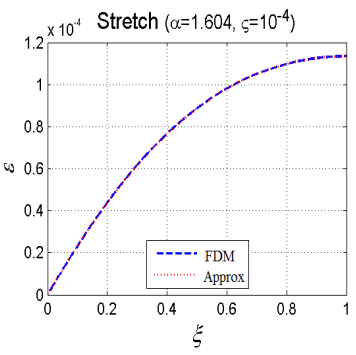

(f)

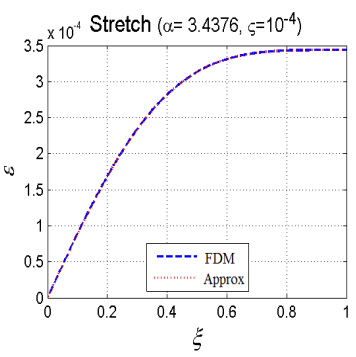

(j)

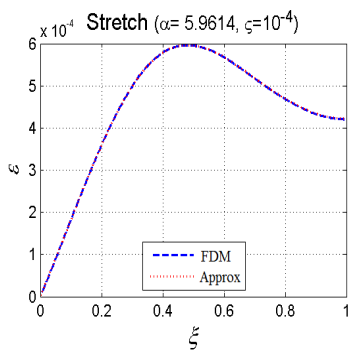

(n)

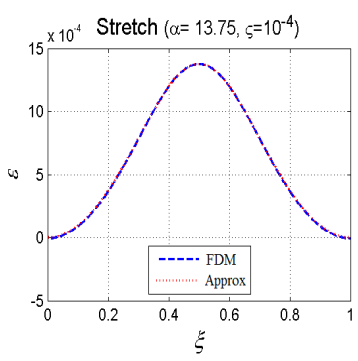

(r)

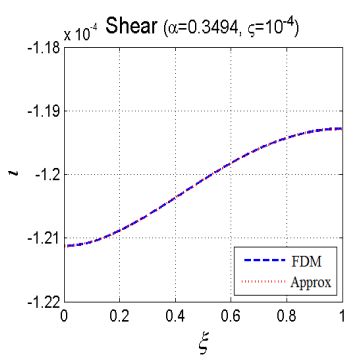

(c)

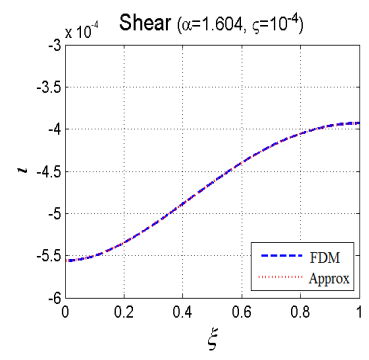

(g)

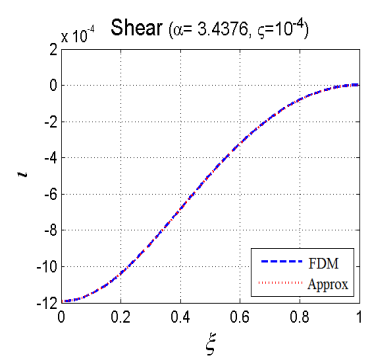

(k)

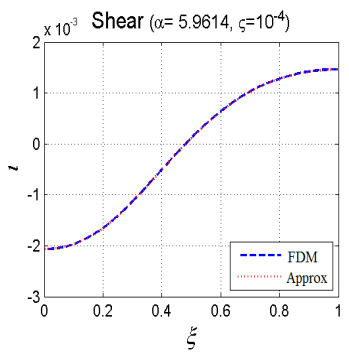

(o)

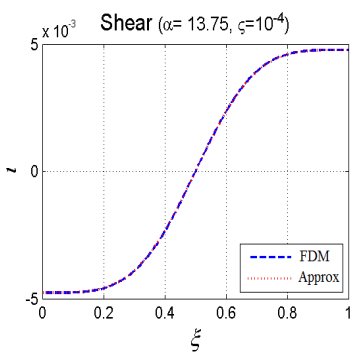

(s)

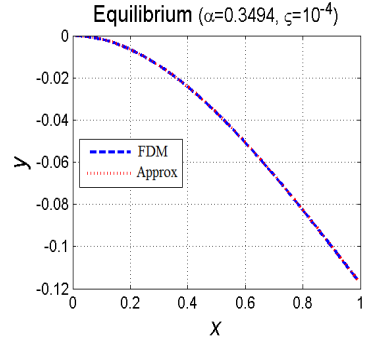

(d)

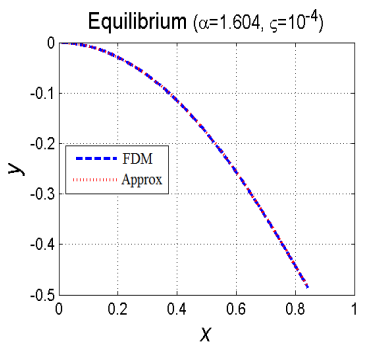

(h)

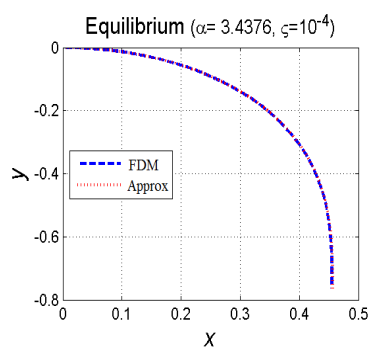

(1)

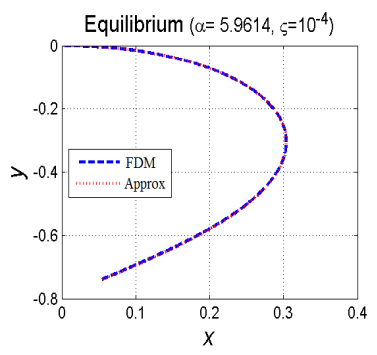

(p)

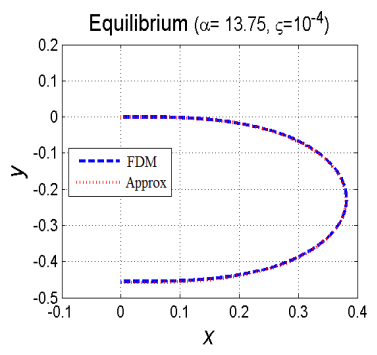

(t)

Fig. 9. Distributions of $\psi, \varepsilon$, and $\imath$ and equilibria of a slender cantilever beam under a transverse force at the free end of the beam, when the slope angle there is: (a) through $(\mathrm{d}),-10^{\circ}$; (e) through $(\mathrm{h}),-45^{\circ}$; (i) through $(1),-90^{\circ}$; (m) through (p), $-135^{\circ}$; and (q) through (t), $-180^{\circ}$, calculated using the FDM (dashed) and the approximate method (dotted). 
and Eq. (14) becomes

$$
-\psi^{\prime \prime}(\xi)=\alpha \sin [\psi(\xi)+\mathfrak{\imath}(\xi)][1+\varepsilon(\xi)], \quad \imath(\xi)=k \varpi \delta \sin [\psi(\xi)+\mathfrak{l}(\xi)][1+\varepsilon(\xi)], \quad \varepsilon(\xi)=-\delta \cos [\psi(\xi)+\mathfrak{\imath}(\xi)]
$$

with boundary conditions

$$
\psi(0)=0, \quad \psi^{\prime}(1)=0
$$

It is easy to verify that the solution $\psi(\xi)=\mathfrak{\imath}(\xi)=0$ and $\varepsilon(\xi)=-\delta$ is a trivial solution of Eq. (42) with boundary conditions in Eq. (43). The question is whether the trivial solution is stable, and if it is unstable, what the stable solution is.

To study the stability of the trivial solution, suppose that there is a perturbation on the solution

$$
\psi(\xi) \rightarrow \psi(\xi)+\tilde{\vartheta}(\xi), \quad \imath(\xi) \rightarrow \mathfrak{l}(\xi)+\tilde{\imath}(\xi), \quad \varepsilon(\xi) \rightarrow \varepsilon(\xi)+\tilde{\varepsilon}(\xi)
$$

and the trivial solution is unstable if there is a non-trivial solution for $\tilde{\vartheta}$, $\tilde{\imath}$, and $\tilde{\varepsilon}$. Substituting Eq. (44) into Eq. (42) yields the linearized equation

$$
\tilde{\vartheta}^{\prime \prime}(\xi)+\alpha(1-\delta)[\tilde{\vartheta}(\xi)+\tilde{\mathfrak{l}}(\xi)]=0, \quad \tilde{\mathfrak{l}}(\xi)=k \varpi \delta(1-\delta)[\tilde{\vartheta}(\xi)+\tilde{\mathfrak{l}}(\xi)], \quad \tilde{\varepsilon}(\xi)=0
$$

One has from the two equations in Eq. (45):

$$
\tilde{\mathfrak{l}}(\xi)=\frac{k \varpi \delta(1-\delta)}{1-k \bar{\omega}(1-\delta)} \tilde{\vartheta}(\xi), \quad \tilde{\vartheta}^{\prime \prime}(\xi)+\bar{\alpha} \tilde{\vartheta}(\xi)=0
$$

where

$$
\bar{\alpha} \equiv \frac{1-\delta}{1-k \varpi \delta(1-\delta)} \alpha
$$

with boundary conditions

$$
\tilde{\vartheta}(0)=0, \quad \tilde{\vartheta}^{\prime}(1)=0
$$

Multiplying both sides of the second equation in Eq. (46) by $\tilde{\vartheta}^{\prime}(\xi)$ and integrating the resulting equation with respect to $\xi$ yield

$$
\left(\frac{d \tilde{\vartheta}(\xi)}{d \xi}\right)^{2}+\bar{\alpha} \tilde{\vartheta}^{2}(\xi)=\bar{\alpha} \tilde{\vartheta}^{2}(1)
$$

The solution of Eq. (49) with boundary conditions in Eq. (48) is

$$
\tilde{\vartheta}(\xi)=\tilde{\vartheta}(1) \sin [\sqrt{\bar{\alpha}} \xi]
$$

where $\bar{\alpha}$ is a solution of

$$
\cos \sqrt{\bar{\alpha}}=0
$$

The minimum solution of Eq. (51) is $\sqrt{\bar{\alpha}}=\frac{\pi}{2}$, which gives $\alpha_{c r}=\frac{\pi^{2}}{4} \frac{1-k \varpi \delta(1-\delta)}{1-\delta}$, and the critical compressive load is $F_{c r}=$ $\alpha_{c r} \frac{E I}{L^{2}}=\frac{\pi^{2}}{4} \frac{1-k \Phi \delta(1-\delta)}{1-\delta} \frac{E I}{L^{2}}$, which is the buckling load of the cantilever beam. Usually, $\delta \ll 1$ and the above buckling load is close to Euler buckling load of a cantilever beam $\bar{F}_{c r}=\frac{\pi^{2}}{4} \frac{E I}{L^{2}}$. 
Suppose $\alpha \geq \alpha_{c r}$, and post-buckling equilibria of the beam can be calculated. If $\delta \ll 1$, an approximate post-buckling equiliribum corresponds to a solution of Eq. (26), which is

$$
\left(\frac{d \bar{\psi}(\xi)}{d \xi}\right)^{2}=2 \alpha[\cos \bar{\psi}(\xi)-\cos \bar{\psi}(1)]
$$

One has from Eq. (52):

$$
\int_{0}^{\bar{\psi}(\xi)} \frac{d \vartheta}{\sqrt{\cos \vartheta-\cos \bar{\psi}(1)}}=\sqrt{2 \alpha \xi}, \quad \int_{0}^{\bar{\psi}(1)} \frac{d \vartheta}{\sqrt{\cos \vartheta-\cos \bar{\psi}(1)}}=\sqrt{2 \alpha}
$$

When $\bar{\psi}(1) \rightarrow 0$, the second equation in Eq. (53) is

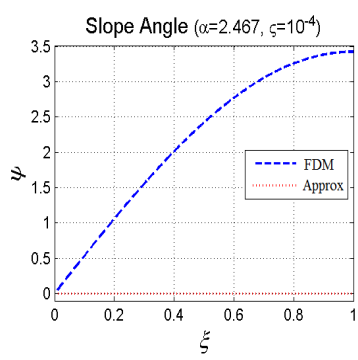

(a)

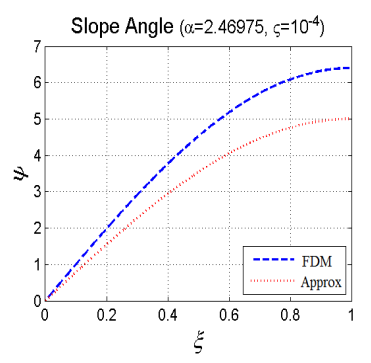

(e)

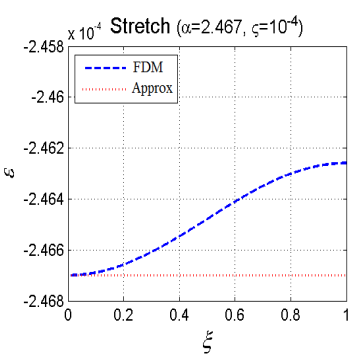

(b)

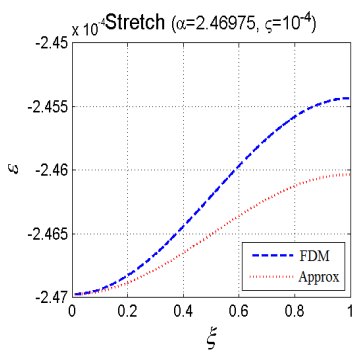

(f)

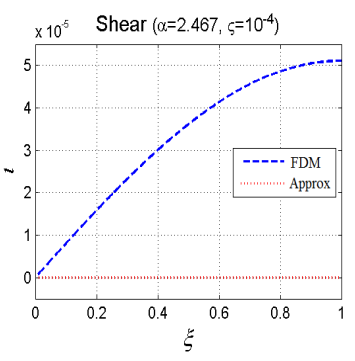

(c)

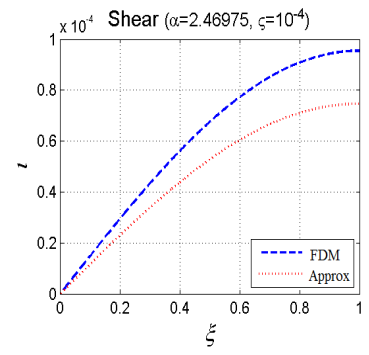

(g)

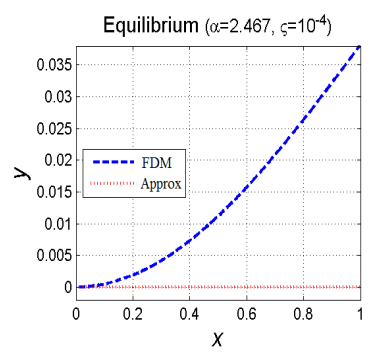

(d)

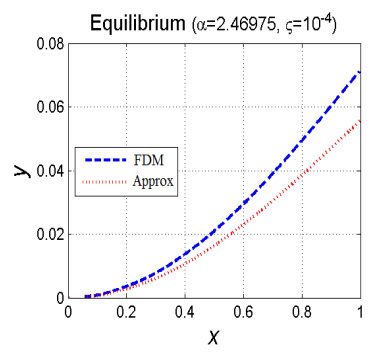

(h)

Fig. 10. Distributions of $\psi, \varepsilon$, and 1 and equilibria of a slender cantilever beam under an axial compressive force, with the dimensionless compressive force: (a) through (d), slightly smaller, and (e) through (h), slightly larger, than Euler buckling load $\bar{\alpha}_{c r}$.

$$
\lim _{x \rightarrow 0} \int_{0}^{x} \frac{d \vartheta}{\sqrt{\cos \vartheta-\cos x}}=\sqrt{2} \lim _{x \rightarrow 0} \int_{0}^{x} \frac{d \vartheta}{\sqrt{x^{2}-\vartheta^{2}}}=\sqrt{2} \lim _{x \rightarrow 0} \int_{0}^{1} \frac{x d \xi}{x \sqrt{1-\xi^{2}}}=\sqrt{2} \int_{0}^{1} \frac{d \xi}{\sqrt{1-\xi^{2}}}=\sqrt{2} \frac{\pi}{2}=\sqrt{2 \bar{\alpha}_{c r}}
$$

where a transformation $\vartheta=x \xi$ has been used. Hence, the perturbation solution does not exist when $\bar{\alpha} \leq \bar{\alpha}_{c r}$, and the trivial solution is the only solution, which is consistent with the result from the buckling analysis. For isotropic materials, $\bar{\omega}=2(1+v)>2$, and for nonuniform shear distributions in cross-sections, $k>1$; hence, one usually has $k \Phi>2$. One has from Eq. (47):

$$
\alpha=\frac{1-k \varpi \delta(1-\delta)}{1-\delta} \bar{\alpha}=\left[1-\left(k \bar{\omega}-\frac{1}{1-\delta}\right) \delta\right] \bar{\alpha}
$$

If $\delta \leq 0.5, \alpha_{c r}$ is slightly smaller than $\bar{\alpha}_{c r}$, which means that the beam buckles before the compressive force reaches Euler buckling load. As shown in Figs. 10(a) - 10(d), when the compressive force $F$ is slightly smaller than Euler buckling load 
$\bar{F}_{c r}$, and correspondingly, $\alpha=2.467$ is slightly smaller than $\bar{\alpha}_{c r}=\frac{\pi^{2}}{4}=2.4674$, the beam buckles but the corresponding Euler-Bernoulli beam does not. When the compressive force $F$ is slightly larger than Euler buckling load, equilibria of the beam are sensitive to the compressive force, as shown in Figs. 10(e) - 10(h), where $\alpha=2.46975$ is only $0.1 \%$ larger than $\bar{\alpha}_{c r}=\frac{\pi^{2}}{4}=2.4674$. Note that there is another post-buckling equilibrium of the beam that bends downward, which is the mirror reflection of the current one.

Distributions of the slope angle and stretch and shear strains of the beam when $\bar{\psi}(1)=45^{\circ}, 90^{\circ}, 135^{\circ}$ are shown in Fig. 11, which correspond to $\alpha=2.6686,3.4376,5.76045$, respectively. One can see that when $\bar{\psi}(1)$ is small, the stretch is

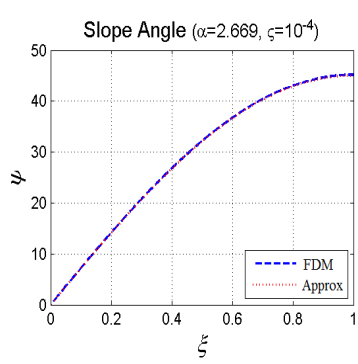

(a)

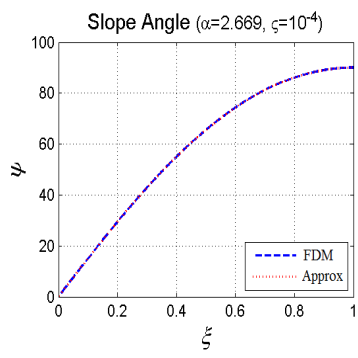

(e)

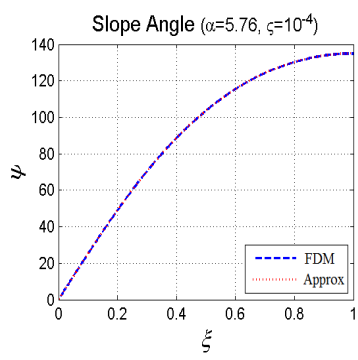

(i)

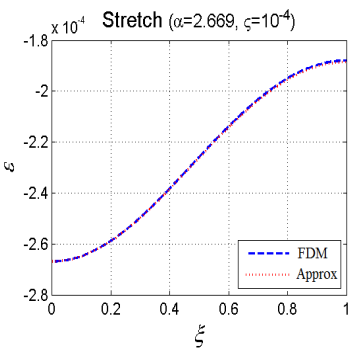

(b)

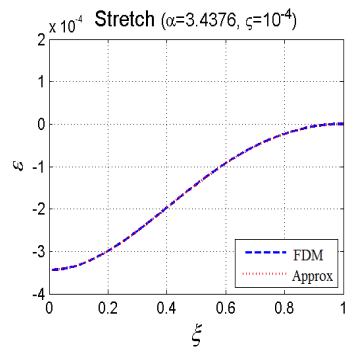

(f)

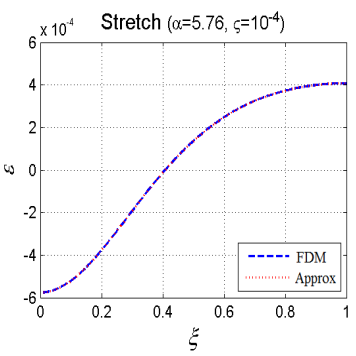

(j)

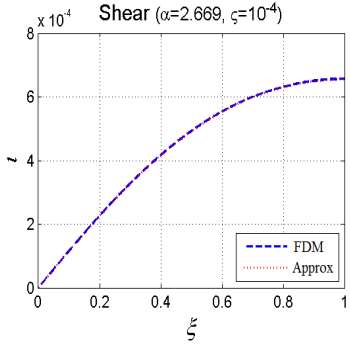

(c)

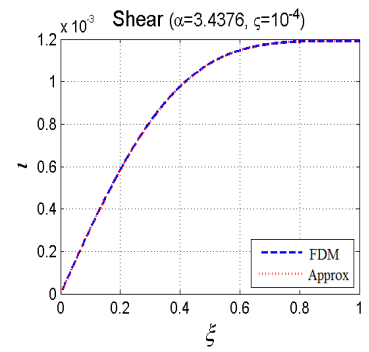

(g)

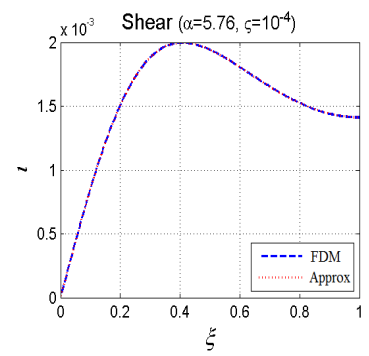

(k)

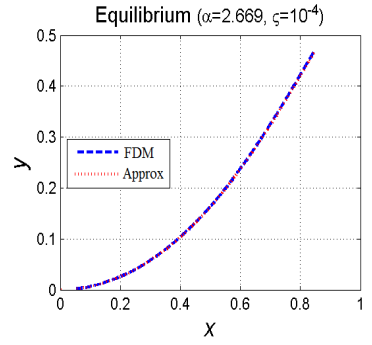

(d)

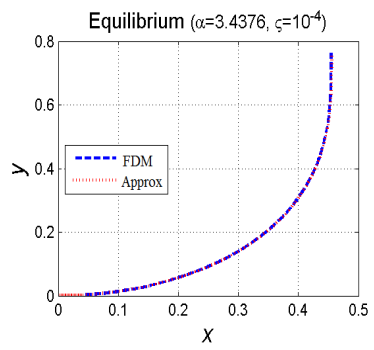

(h)

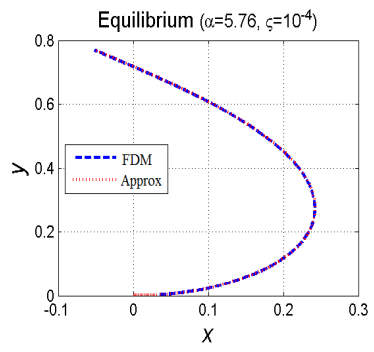

(1)

Fig. 11. Distributions of $\psi, \varepsilon$, and $\mathrm{l}$, and equilibria of a slender cantilever beam under an axial compressive force, when the slope angle at the free end is: (a) through (d), $45^{\circ}$; (e) through (h), $90^{\circ}$; and (i) through (l), $135^{\circ}$, calculated using the FDM (dashed) and the approximate method (dotted).

almost uniform along the beam, as shown in Fig. 11(b). When $\bar{\psi}(1)=90^{\circ}$, there is almost no stretch at the free end, and the stretch at the fixed end is significant, as shown in Fig. 11(f); when $\bar{\psi}(1)=135^{\circ}$, there are a compression near the fixed end and an extension near the free end, as shown in Fig. 11(j). Profiles of the slope angle in Figs. 10(e), 11(a), 11(e), and 11(i) are very close to $\bar{\psi}(1) \sin \frac{\pi \xi}{2}$, which is the perturbation solution in Eq. (50). It is worth mentioning that there are higher-order buckling solutions with complex configurations as shown in Fig. 12, because Eq. (51) has other solutions

$$
\bar{\alpha}=\left(\frac{3 \pi}{2}\right)^{2}=22.2066, \quad \bar{\alpha}=\left(\frac{5 \pi}{2}\right)^{2}=61.6850, \quad \cdots,
$$

and the corresponding perturbation solutions are

$$
\psi(\xi)=\bar{\psi}(1) \sin \frac{3 \pi \xi}{2}, \quad \psi(\xi)=\bar{\psi}(1) \sin \frac{5 \pi \xi}{2}, \quad \cdots
$$


These solutions are possible only for long slender beams. Distributions of the slope angle and stretch and shear strains, and

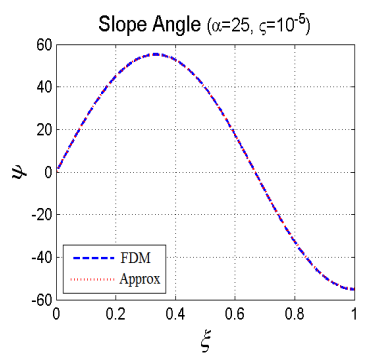

(a)

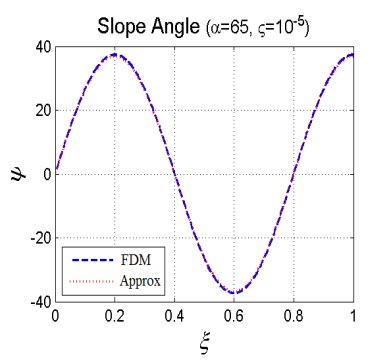

(e)

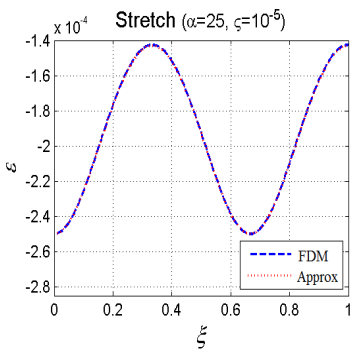

(b)

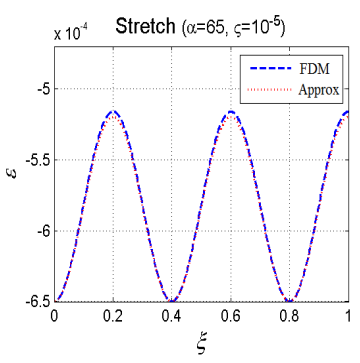

(f)

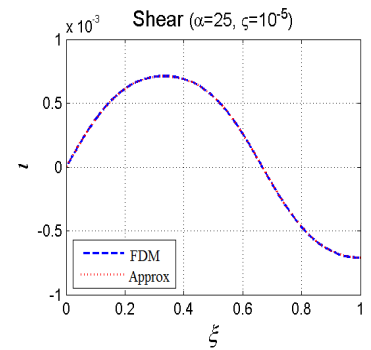

(c)

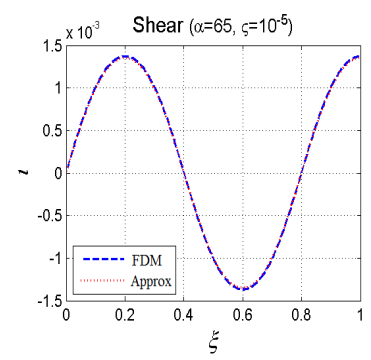

(g)

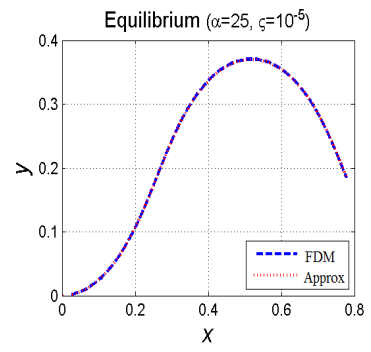

(d)

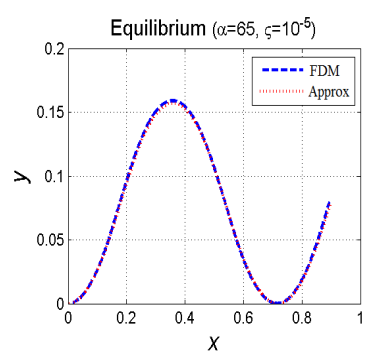

(h)

Fig. 12. Distributions of $\psi, \varepsilon$, and $\mathrm{l}$, and equilibria of a long slender cantilever beam under an axial compressive force, when the dimensionless force $\alpha$ is: (a) through (d), 25; and (e) through (h), 65, calculated using the FDM (dashed) and the approximate method (dotted).

the equilibrium in Fig. 12(a) - 12(d), respectively, correspond to $\alpha=25$, which is slightly larger than the second smallest solution of Eq. (51), and those in Fig. 12(e) - 12(h) correspond to $\alpha=65$, which is slightly larger than the third smallest solution of Eq. (51). The slope angle distributions in Figs. 12(a) and 12(e) are similar to the perturbation solutions in Eq. (57).

\section{Buckling of Uniform Beams}

Consider equilibria of beams with various boundary conditions under an axial compressive force $F$, as shown in Fig. 13 . The vertical displacement of the beam at $s=L$ vanishes in all the cases:

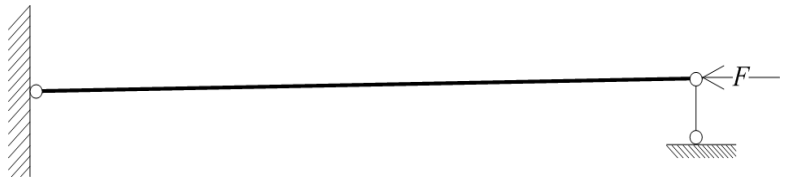

(a)

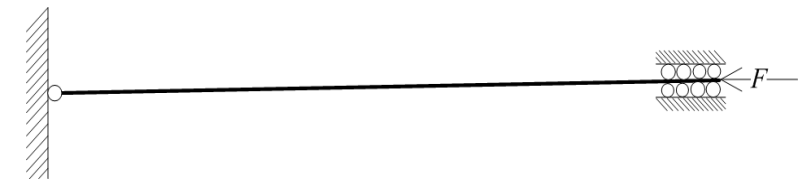

(c)

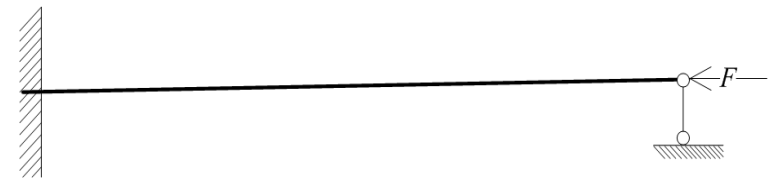

(b)

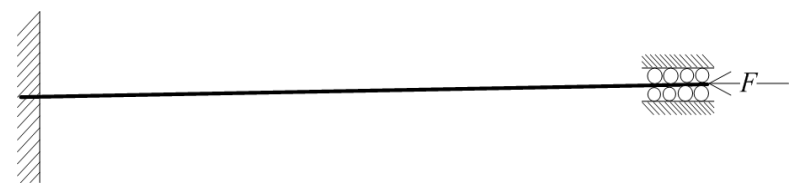

(d)

Fig. 13. Schematics of beams under axial compressive forces, with (a) pinned-pinned, (b) fixed-pinned, (c) pinned-fixed, and (d) fixed-fixed boundary conditions.

$$
\mathcal{C} \equiv y(L)=\int_{0}^{L} \sin \theta(s)[1+\varepsilon(s)] d s=\int_{0}^{L}\{\sin [\phi(s)+\gamma(s)][1+\varepsilon(s)]\} d s=0,
$$


which gives a constraint on dependent variables. Static equations of equilibria of the beams can be derived using the principle of virtual work with constraints $\delta \mathcal{P}=\delta \mathcal{W}+\lambda \delta \mathcal{C}$, where $\lambda$ is a Lagrangian multiplier, which yields

$$
\begin{gathered}
-\left(E I(s) \phi^{\prime}(s)\right)^{\prime}=\{F \sin [\phi(s)+\gamma(s)]-\lambda \cos [\phi(s)+\gamma(s)]\}[1+\varepsilon(s)]=\frac{G A(s)}{k} \gamma(s), \\
E A(s) \varepsilon(s)=-F \cos [\phi(s)+\gamma(s)]-\lambda \sin [\phi(s)+\gamma(s)],
\end{gathered}
$$

where $\lambda$ is the vertical constraint force. The pinned-pinned boundary conditions for the case in Fig. 13(a) are

$$
E I(0) \theta^{\prime}(0)=0, \quad E I(L) \theta^{\prime}(L)=0
$$

The fixed-pinned or pined-fixed boundary conditions for the cases in Fig. 13(b) or 13(c) are:

$$
\theta(0)=0, \quad E I(0) \theta^{\prime}(L)=0 ; \quad \text { or } \quad E I(0) \theta^{\prime}(0)=0, \quad \theta(L)=0 .
$$

The fixed-fixed boundary conditions for the case in Fig. 13(d) are

$$
\theta(0)=0, \quad \theta(L)=0
$$

Consider uniform beams and let $\mu=\frac{\lambda}{F}$. Equations (58) and (59) become

$$
-\psi^{\prime \prime}(\xi)=\alpha\{\sin [\psi(\xi)+\mathfrak{l}(\xi)]-\mu \cos [\psi(\xi)+\mathfrak{l}(\xi)]\}[1+\varepsilon(\xi)], \quad \varepsilon(\xi)=-\delta\{\mu \sin [\psi(\xi)+\mathfrak{l}(\xi)]+\cos [\psi(\xi)+\mathfrak{l}(\xi)]\},
$$

$$
\mathfrak{l}(\xi)=k \varpi \delta\{\sin [\psi(\xi)+\mathfrak{l}(\xi)]-\mu \cos [\psi(\xi)+\mathfrak{l}(\xi)]\}[1+\varepsilon(\xi)], \quad \int_{0}^{1} \sin [\psi(\xi)+\mathfrak{l}(\xi)][1+\varepsilon(\xi)] d \xi=0
$$

\subsection{Buckling Analysis}

It is obvious that $\psi(\xi)=\mathfrak{l}(\xi)=0, \varepsilon(\xi)=-\delta$, and $\mu=0$ are a trivial solution of Eqs. (63) and (64). To determine the stability of the trivial solution, suppose that there is a perturbation on this solution

$$
\psi(\xi) \rightarrow \psi(\xi)+\tilde{\vartheta}(\xi), \quad \varepsilon(\xi) \rightarrow \varepsilon(\xi)+\tilde{\varepsilon}(\xi), \quad \imath(\xi) \rightarrow \mathfrak{\imath}(\xi)+\tilde{\mathfrak{l}}(\xi), \quad \mu \rightarrow \mu-\tilde{\mu}
$$

Linearized equations of Eqs. (63) and (64) are

$$
\tilde{\vartheta}^{\prime \prime}(\xi)+\alpha(1-\delta)[\tilde{\vartheta}(\xi)+\tilde{\mathfrak{l}}(\xi)+\tilde{\mu}]=0, \quad \tilde{\varepsilon}(\xi)=0, \quad \tilde{\mathfrak{l}}(\xi)=k \varpi \delta(1-\delta)[\tilde{\vartheta}(\xi)+\tilde{\mathfrak{l}}(\xi)+\tilde{\mu}], \quad \int_{0}^{1} \tilde{\vartheta}(\xi) d \xi=0 .
$$

One has from the first and third equations in Eq. (66):

$$
\tilde{\mathfrak{i}}(\xi)=\frac{k \varpi \delta(1-\delta)}{1-k \varpi \delta(1-\delta)}[\tilde{\vartheta}(\xi)+\tilde{\mu}], \quad \tilde{\vartheta}^{\prime \prime}(\xi)+\bar{\alpha}[\tilde{\vartheta}(\xi)+\tilde{\mu}]=0
$$

where the constant $\bar{\alpha}$ is the same as that in Eq. (47); if $\delta \ll 1$, one has $\bar{\alpha} \approx \alpha$. Multiplying both sides of the second equation in Eq. (67) by $\tilde{\vartheta}^{\prime}(\xi)$ and integrating the resulting equation with respect to $\xi$ yield

$$
\left(\frac{d \tilde{\vartheta}(\xi)}{d \xi}\right)^{2}+\bar{\alpha}[\tilde{\vartheta}(\xi)+\tilde{\mu}]^{2}=\text { const }
$$




\subsubsection{Pinned-Pinned Boundary Conditions}

Consider the pinned-pinned boundary conditions in Eq. (60):

$$
\tilde{\vartheta}^{\prime}(0)=\tilde{\vartheta}^{\prime}(1)=0
$$

One has from Eqs. (68) and (69):

$$
\left(\frac{d \tilde{\vartheta}(\xi)}{d \xi}\right)^{2}+\bar{\alpha}[\tilde{\vartheta}(\xi)+\tilde{\mu}]^{2}=\bar{\alpha}[\tilde{\vartheta}(0)+\tilde{\mu}]^{2}, \quad[\tilde{\vartheta}(0)+\tilde{\mu}]^{2}=[\tilde{\vartheta}(1)+\tilde{\mu}]^{2}, \quad \int_{0}^{1} \tilde{\vartheta}(\xi) d \xi=0
$$

The first equation in Eq. (70) gives

$$
\int_{\tilde{\vartheta}(0)}^{\tilde{\vartheta}(\xi)} \frac{d \tilde{\vartheta}}{\sqrt{[\tilde{\vartheta}(0)+\tilde{\mu}]^{2}-(\tilde{\vartheta}+\tilde{\mu})^{2}}}=\int_{\tilde{\vartheta}(0)+\tilde{\mu}}^{\tilde{\vartheta}(\xi)+\tilde{\mu}} \frac{d x}{\sqrt{[\tilde{\vartheta}(0)+\tilde{\mu}]^{2}-x^{2}}}=-\sin ^{-1} \frac{\tilde{\vartheta}(\xi)+\tilde{\mu}}{\tilde{\vartheta}(0)+\tilde{\mu}}+\frac{\pi}{2}=\sqrt{\bar{\alpha} \xi}
$$

which is equivalent to

$$
\tilde{\vartheta}(\xi)+\tilde{\mu}=(\tilde{\vartheta}(0)+\tilde{\mu}) \cos [\sqrt{\bar{\alpha}} \xi]
$$

For the second and third equations in Eq. (70) to be satisfied, it is required that

$$
\sqrt{\bar{\alpha}}=k \pi, \quad \tilde{\mu}=0 .
$$

This indicates that the minimum solution of the first equation in Eq. (73) is $\alpha_{c r}=\pi^{2} \frac{1-k \Phi \delta(1-\delta)}{1-\delta}$, and the critical compressive load is $F_{c r}=\frac{\alpha_{c r} E I}{L^{2}}=\pi^{2} \frac{1-k \omega \delta(1-\delta)}{1-\delta} \frac{E I}{L^{2}}$. Usually, $\delta$ is small and the above buckling load is close to Euler buckling load $\bar{F}_{c r}=\frac{\pi^{2} E I}{L^{2}}$. The corresponding perturbation solution is

$$
\tilde{\vartheta}(\xi)=\tilde{\vartheta}(0) \cos (k \pi \xi), \quad \tilde{\mu}=0, \quad \tilde{\varepsilon}(\xi)=0 .
$$

\subsubsection{Fixed-Pinned Boundary Conditions}

Consider the fixed-pinned boundary conditions in Eq. (61):

$$
\tilde{\vartheta}(0)=\tilde{\vartheta}^{\prime}(1)=0
$$

One has from Eqs. (68) and (75):

$$
\left(\frac{d \vartheta(\xi)}{d \xi}\right)^{2}+\bar{\alpha}[\tilde{\vartheta}(\xi)+\tilde{\mu}]^{2}=\bar{\alpha}[\tilde{\vartheta}(1)+\tilde{\mu}]^{2}
$$

Equations (75) and (76) give

$$
\int_{\tilde{\vartheta}(\xi)}^{\tilde{\vartheta}(1)} \frac{d \tilde{\vartheta}}{\sqrt{[\tilde{\vartheta}(1)+\tilde{\mu}]^{2}-(\tilde{\vartheta}+\tilde{\mu})^{2}}}=\int_{\tilde{\vartheta}(\xi)+\tilde{\mu}}^{\tilde{\vartheta}(1)+\tilde{\mu}} \frac{d x}{\sqrt{[\tilde{\vartheta}(1)+\tilde{\mu}]^{2}-x^{2}}}=\frac{\pi}{2}-\sin ^{-1} \frac{\tilde{\vartheta}(\xi)+\tilde{\mu}}{\tilde{\vartheta}(1)+\tilde{\mu}}=\sqrt{\bar{\alpha}}(1-\xi)
$$

which is equivalent to

$$
\tilde{\vartheta}(\xi)=(\tilde{\vartheta}(1)+\tilde{\mu}) \cos [\sqrt{\bar{\alpha}}(1-\xi)]-\tilde{\mu} .
$$


Substituting Eq. (75) into Eq. (78) yields

$$
\frac{\tilde{\vartheta}(1)+\tilde{\mu}}{\sqrt{\bar{\alpha}}} \sin \sqrt{\bar{\alpha}}=\tilde{\mu}, \quad(\tilde{\vartheta}(1)+\tilde{\mu}) \cos \sqrt{\bar{\alpha}}=\tilde{\mu} .
$$

Solving Eq. (79) yields

$$
\tan \sqrt{\bar{\alpha}}=\sqrt{\bar{\alpha}}, \quad \tilde{\mu}=\frac{\cos \sqrt{\bar{\alpha}}}{1-\cos \sqrt{\bar{\alpha}}} \tilde{\vartheta}(1)
$$

The minimum solution of the first equation in Eq. (80) is $\sqrt{\bar{\alpha}}=4.4934$, which gives $\alpha_{c r}=4.4934^{2} \frac{1-k \omega \delta(1-\delta)}{1-\delta}=\frac{\pi^{2}}{K^{2}} \frac{1-k \omega \delta(1-\delta)}{1-\delta}$, where $K=0.6992$, and the critical compressive load is $F_{c r}=\frac{\pi^{2}}{K^{2}} \frac{1-k \Phi \delta(1-\delta)}{1-\delta} \frac{E I}{L^{2}}$. When $\delta$ is small, it is close to Euler buckling load $\bar{F}_{c r}=\frac{\pi^{2}}{K^{2}} \frac{E I}{L^{2}}$.

\subsubsection{Fixed-Fixed Boundary Conditions}

Consider the fixed-fixed boundary conditions in Eq. (62):

$$
\tilde{\vartheta}(0)=\tilde{\vartheta}(1)=0
$$

The constant in Eq. (68) is nonnegative and can be denoted by $\Omega^{2}$. One has from Eqs. (68) and (81):

$$
\int_{0}^{\tilde{\vartheta}(\xi)} \frac{d \tilde{\vartheta}}{\sqrt{\Omega^{2}-(\tilde{\vartheta}+\tilde{\mu})^{2}}}=\int_{\tilde{\mu}}^{\tilde{\vartheta}(\xi)+\tilde{\mu}} \frac{d x}{\sqrt{\Omega^{2}-x^{2}}}=\sin ^{-1} \frac{\tilde{\vartheta}(\xi)+\tilde{\mu}}{\Omega}-\sin ^{-1} \frac{\tilde{\mu}}{\Omega}=\sqrt{\bar{\alpha}} \xi
$$

Let $\phi=\sin ^{-1} \frac{\tilde{\mu}}{\Omega} ;$ Eq. (82) gives

$$
\tilde{\vartheta}(\xi)=\Omega\{\sin [\sqrt{\bar{\alpha}} \xi+\phi]-\sin \phi\}
$$

Substituting Eq. (83) into the second equation in Eq. (66) and Eq. (81) yields

$$
\cos \phi-\cos (\sqrt{\bar{\alpha}}+\phi)=\sqrt{\bar{\alpha}} \sin \phi, \quad \sin (\sqrt{\bar{\alpha}}+\phi)=\sin \phi,
$$

which is equivalent to

$$
\frac{1-\cos \sqrt{\bar{\alpha}}}{\sqrt{\bar{\alpha}}-\sin \sqrt{\bar{\alpha}}}=\tan \phi=\frac{\sin \sqrt{\bar{\alpha}}}{1-\cos \sqrt{\bar{\alpha}}}
$$

Solving Eq. (85) yields

$$
\sin \frac{\sqrt{\bar{\alpha}}}{2}\left(\frac{\sqrt{\bar{\alpha}}}{2} \cos \frac{\sqrt{\bar{\alpha}}}{2}-\sin \frac{\sqrt{\bar{\alpha}}}{2}\right)=0
$$

which indicates that either $\sqrt{\bar{\alpha}}=2 \mathrm{k} \pi$, where $\mathrm{k}=1,2, \cdots$, or $\tan \frac{\sqrt{\bar{\alpha}}}{2}=\frac{\sqrt{\bar{\alpha}}}{2}$, and correspondingly, $\phi=0$ or $\frac{\pi-\sqrt{\bar{\alpha}}}{2}$. The minimum solution of $\sqrt{\bar{\alpha}}$ is $2 \pi$; consequently, $\alpha_{c r}=4 \pi^{2} \frac{1-k \varpi \delta(1-\delta)}{1-\delta}=\left(\frac{\pi}{K}\right)^{2} \frac{1-k \varpi \delta(1-\delta)}{1-\delta}$, where $K=0.5$; hence, the critical compressive load is $F_{c r}=\left(\frac{\pi}{K}\right)^{2} \frac{1-k \omega \delta(1-\delta)}{1-\delta} \frac{E I}{L^{2}}$. When $\delta$ is small, it is close to Euler buckling load $\bar{F}_{c r}=\frac{\pi^{2}}{K^{2}} \frac{E I}{L^{2}}$. The $(2 \mathrm{k}-1)$ th 
solution of Eq. (86) is $\bar{\alpha}=4 \mathrm{k}^{2} \pi^{2}$; by the first equation in Eq. (84), one has $\phi=0$, and the corresponding perturbation solution is

$$
\tilde{\vartheta}(\xi)=\Omega \sin (2 \mathrm{k} \pi \xi)
$$

The $2 \mathrm{kth}$ solution of Eq. (86) is the kth solution of

$$
\tan \frac{\sqrt{\bar{\alpha}}}{2}=\frac{\sqrt{\bar{\alpha}}}{2}
$$

one has from Eq. (85):

$$
\phi=\frac{\pi}{2}-\frac{\sqrt{\bar{\alpha}}}{2}
$$

and the corresponding perturbation solution is

$$
\tilde{\vartheta}(\xi)=\Omega\left\{\cos \left[\sqrt{\bar{\alpha}}\left(\xi-\frac{1}{2}\right)\right]-\cos \frac{\sqrt{\bar{\alpha}}}{2}\right\} .
$$

\subsection{Numerical Solution of Equilibria}

When $\alpha>\alpha_{c r}$, the trivial solution is not stable any more. One needs to directly solve the nonlinear equations in Eqs. (63) and (64) to find their nontrivial solutions. It is difficult to analytically solve them, but they can be numerically solved as follows:

1. Partition the unit length of the beam into $\mathrm{N}$ elements using nodes $s_{n}=\frac{n}{N}$, where $n=0,1, \cdots, N$, with the element length $h=\frac{1}{N}$.

2. Discretize Eqs. (63) and (64) using central difference to yield

$$
\begin{aligned}
& N^{2} \psi_{n-1}-2 N^{2} \psi_{n}+\alpha\left[\sin \left(\psi_{n}+\mathfrak{l}_{n}\right)-\mu \cos \left(\psi_{n}+\mathfrak{l}_{n}\right)\right]\left(1+\varepsilon_{n}\right)+N^{2} \psi_{n+1}=0, \quad n=1,2, \cdots, N-1, \\
& \mathbf{l}_{n}-k \varpi \delta\left[\sin \left(\psi_{n}+\mathbf{l}_{n}\right)-\mu \cos \left(\psi_{n}+\mathbf{l}_{n}\right)\right]\left(1+\varepsilon_{n}\right)=0, \quad n=0,1, \cdots, N \\
& \varepsilon_{n}+\delta\left[\mu \sin \left(\psi_{n}+\mathbf{l}_{n}\right)+\cos \left(\psi_{n}+\mathbf{l}_{n}\right)\right]=0, \quad n=0,1, \cdots, N, \\
& \frac{1}{2} \sin \left(\psi_{0}+\mathfrak{\imath}_{0}\right)\left(1+\varepsilon_{0}\right)+\sum_{n=1}^{N-1} \sin \left(\psi_{n}+\mathbf{l}_{n}\right)\left(1+\varepsilon_{n}\right)+\frac{1}{2} \sin \left(\psi_{N}+\mathbf{l}_{N}\right)\left(1+\varepsilon_{N}\right)=0
\end{aligned}
$$

which are $3 N+2$ algebraic equations with $3 N+4$ unknowns $\psi_{n}, \varepsilon_{n}, \mathbf{1}_{n}$, and $\mu$, where $n=0,1, \cdots, N$.

3. The other two equations can be derived from the boundary conditions:

(a) pinned-pinned:

$$
3 \psi_{0}-4 \psi_{1}+\psi_{2}=0, \quad-\psi_{N-2}+4 \psi_{N-1}-3 \psi_{N}=0
$$

(b) fixed-pinned:

$$
\psi_{0}=0, \quad-\psi_{N-2}+4 \psi_{N-1}-3 \psi_{N}=0
$$


(c) fixed-fixed:

$$
\psi_{0}=0, \quad \psi_{N}=0
$$

4. Solving Eqs. (91) - (94) using one of the boundary conditions in Eqs. (95) - (97), one can accurately calculate equilibria of the beam.

Distributions of the slope angle and stretch and shear strains, as well as post-buckling equilibria of a pinned-pinned beam, a fixed-pinned beam, and a fixed-fixed beam are shown in Figs. 14, 15, and 16, respectively; the corresponding $\alpha$ and $\zeta$ are shown in each figure, and $\delta=\alpha \varsigma$ as shown in Eq. (12). In all the examples, the beams are slender with $\varsigma=10^{-4}$ or $10^{-5}$, and the results calculated from the FDM and the approximate method agree well. As discussed in Sec. 5.1, the dimensionless critical buckling loads for pinned-pinned, fixed-pinned, and fixed-fixed Euler-Bernoulli beams are $\bar{\alpha}_{c r}=\pi^{2}=9.8696, \bar{\alpha}_{c r}=20.19073$, and $\bar{\alpha}_{c r}=4 \pi^{2}=39.4784$, respectively. The shear correction factor $k=1.3$, and $\varpi=\frac{E}{G}=2.6$; the buckling loads $\alpha_{c r}$ in Eq. (55) are slightly smaller than $\bar{\alpha}_{c r}$ in all the cases and the calculated equilibria are slightly different, as shown in Figs. 14(a) - 14(d), 15(a) - 15(d), and 16(a) - 16(d). The equilibria are sensitive to compressive forces near critical compressive loads, as shown in Figs. 14(e) - 14(h), 15(e) - 15(h), and 16(e) - 16(h). When the compressive forces are much larger than the critical compressive loads, the approximate solutions get closer to the solutions from the FDM, as shown in Figs. 14(i) - 14(1), 15(i) - 15(1), and 16(i) - 16(l). When the compressive forces become larger, there can exist higher-order buckling modes with complex configurations. The second-order buckling modes are shown in Figs. 14(m) - 14(p), 15(m) - 15(p), and 16(m) - 16(p), and the third-order buckling modes are shown in Figs. 14(q) - 14(t), 15(q) - 15(t), and 16(q) - 16(t). As one can see, the fixed-fixed beam is stiffer than the fixed-pinned beam, since the critical buckling load in the former is larger than that in the latter, and the fixed-pinned beam is stiffer than the pinned-pinned beam, which are consistent with intuition.

\section{Conclusion}

The current formulation is derived from a geometrically exact analysis of beam deformations, and can be applied to both slender and thick planar beams. The shear strain is taken as a dependent variable, which does not cause shear locking in the formulation. When a beam is slender, the nonlinear Euler-Bernoulli beam formulation, where only the bending deformation is taken into account, and stretch and shear strains of the beam can be subsequently estimated from it, is shown to be a good approximation of the current formulation. Relations between end forces and moments and deformations of the beam can be easily calculated from both formulations, which can be useful in the study of compliant mechanisms. The nonlinear buckling beam problems for not only first-order buckling modes, but also higher-order ones, can be studied using the current formulation, and post-buckling equilibria can be accurately calculated. The critical buckling loads calculated from the current formulation are shown to be slightly smaller than those from the Euler-Bernoulli beam formulation. The current formulation can be extended to curved beams, and their equilibria, buckling modes, and post-buckling equilibria can be studied using similar procedures.

\section{Achnowledgements}

This work is supported by the MSC Software Corporation, the National Science Foundation through Grant No. CMMI1000830, and the National Science Foundation of China through Grant No. 11442006. The first author would like to thank Jose Ortiz for his help. The third author would like to thank the China Scholarship Council for supporting his visit to the University of Maryland, Baltimore County.

\section{References}

[1] Love, A. E. H., 1944, A Treatise on the Mathematical Theory of Elasticity, Dover Publications, New York, pp. 381-454.

[2] Antman, S. S., 2005, Nonlinear Problems of Elasticity, Springer, New York, pp. 261-358.

[3] Howell, L. L., 2001, Compliant Mechanisms, Wiley-Interscience, New York.

[4] Howell L L, and Midha A, 1995, "Parametric deflection approximations for end-loaded, large-deflection beams in compliant mechanisms", Journal of Mechanical Design, 117(1), pp. 156-165.

[5] Su H J, 2009, "A pseudorigid-body 3R model for determining large deflection of cantilever beams subject to tip loads", Journal of Mechanisms and Robotics, 1(2), pp. 021008.

[6] J.-S. Duan, R. Rach, and A.-M. Wazwaz, 2013, "Solution of the model of beam-type micro-and nano-scale electrostatic actuators by a new modified Adomian decomposition method for nonlinear boundary value problems", International Journal of Non-Linear Mechanics, 49, pp. 159-169. 


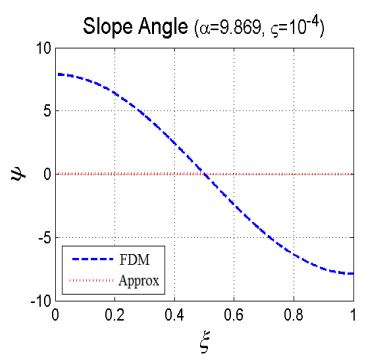

(a)

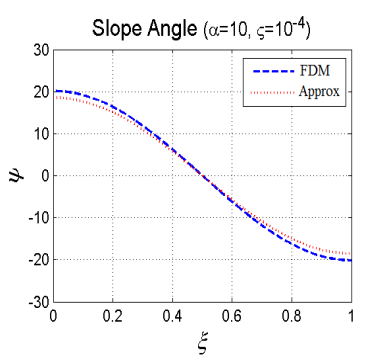

(e)

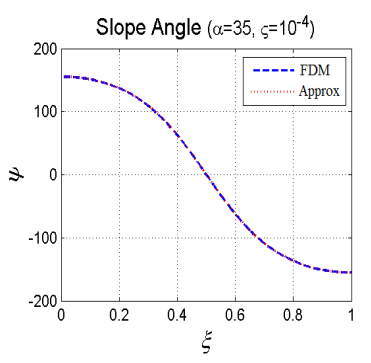

(i)

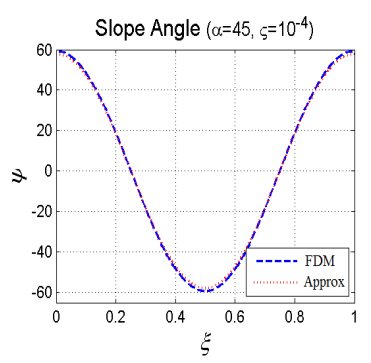

(m)

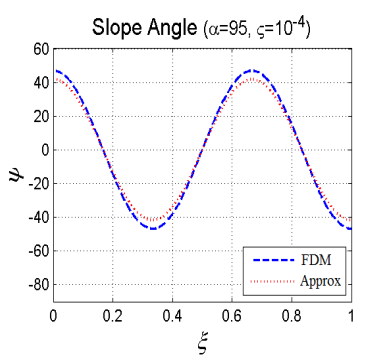

(q)

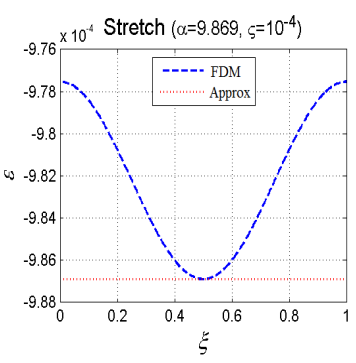

(b)

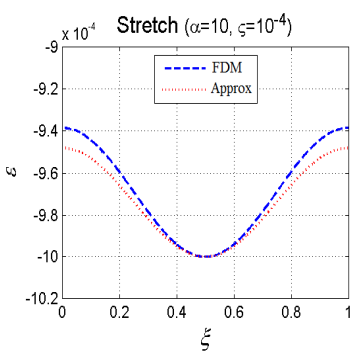

(f)

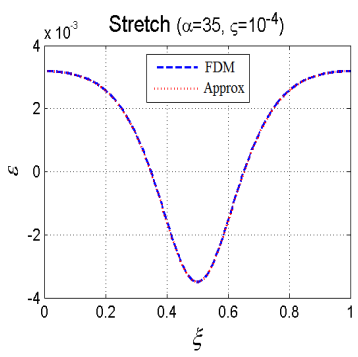

(j)

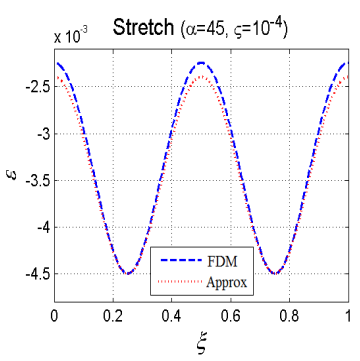

(n)

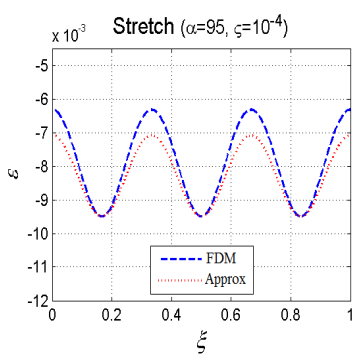

(r)

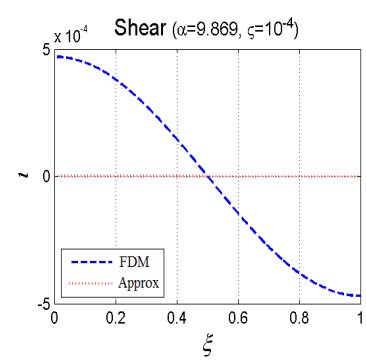

(c)

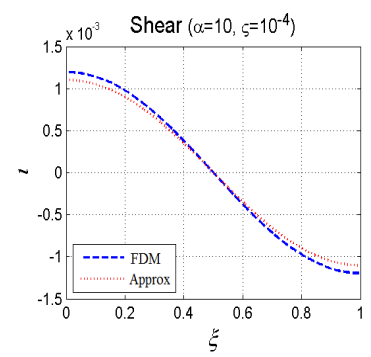

(g)

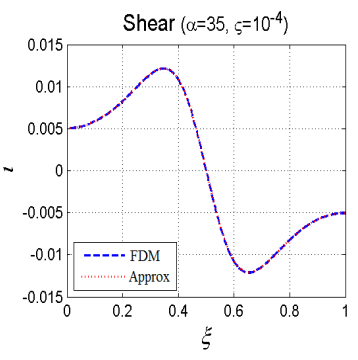

(k)

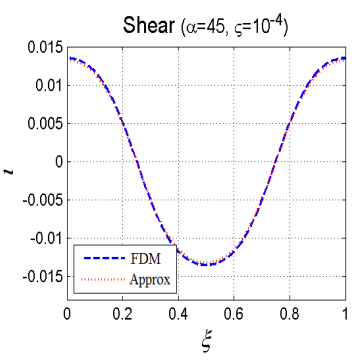

(o)

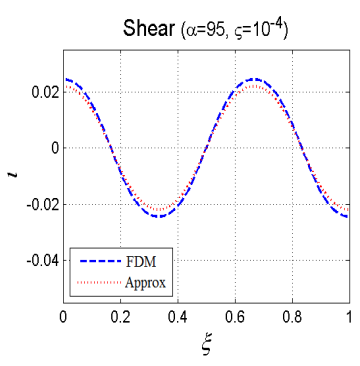

(s)

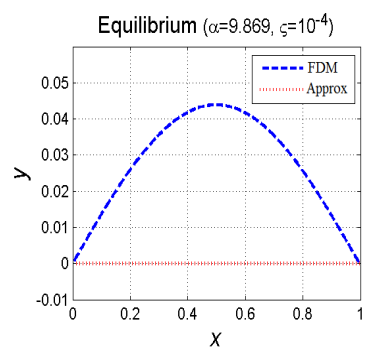

(d)

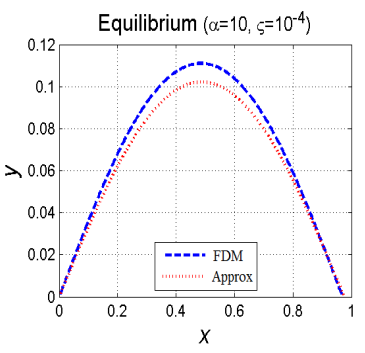

(h)

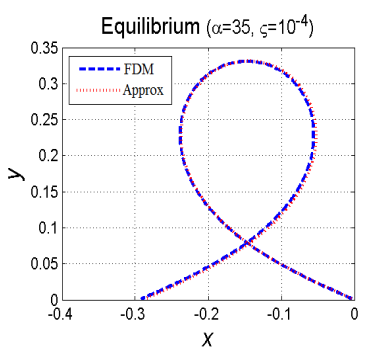

(1)

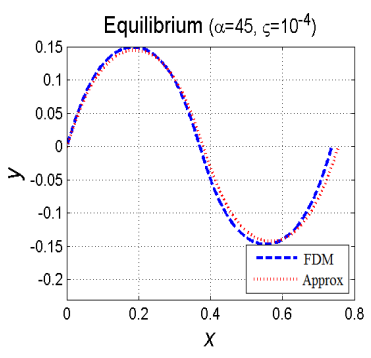

(p)

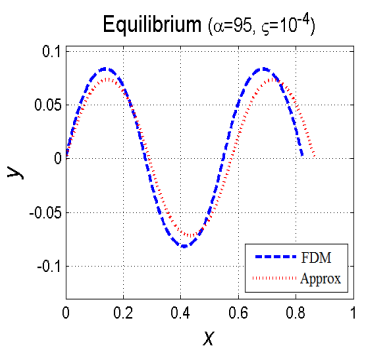

(t)

Fig. 14. Distributions of $\psi, \varepsilon$, and $\imath$ and equilibria of a pinned-pinned beam under an axial compressive force, when the dimensionless compressive force $\alpha$ is: (a) through (d), 9.869; (e) through (h), 10; (i) through (l), 35; (m) through (p), 45; and (q) through (s), 95, calculated using the FDM (dashed) and the approximate method (dotted). 


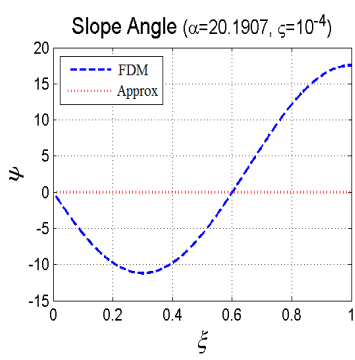

(a)

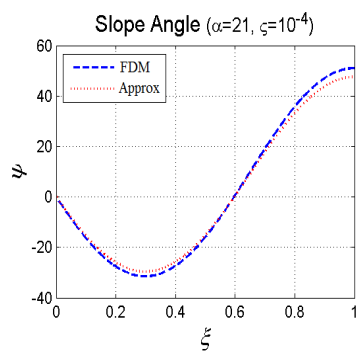

(e)

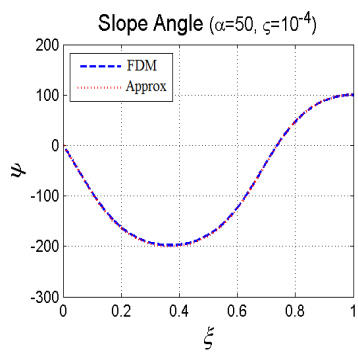

(i)

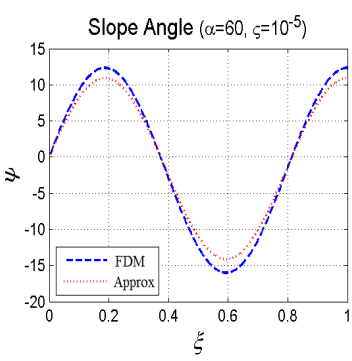

(m)

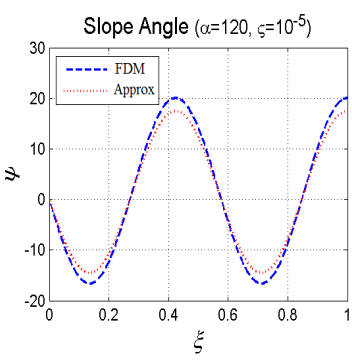

(q)

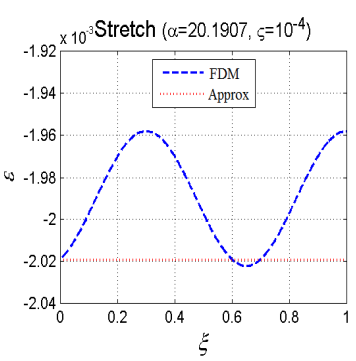

(b)

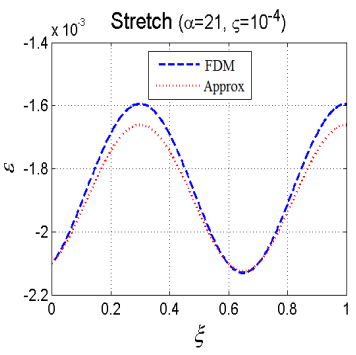

(f)

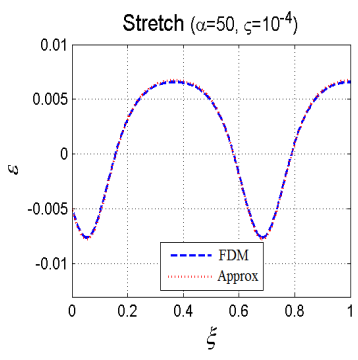

(j)

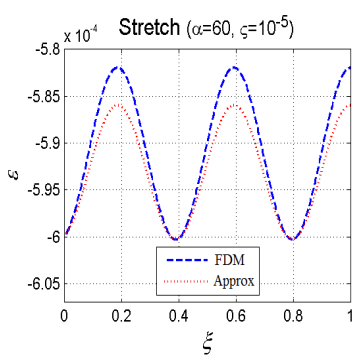

(n)

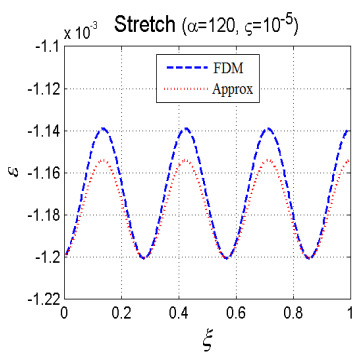

(r)

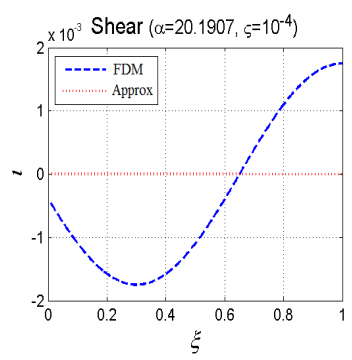

(c)

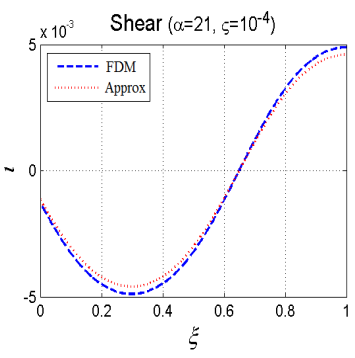

(g)

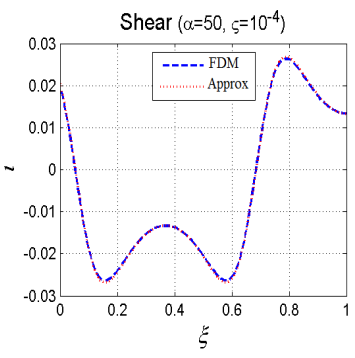

(k)

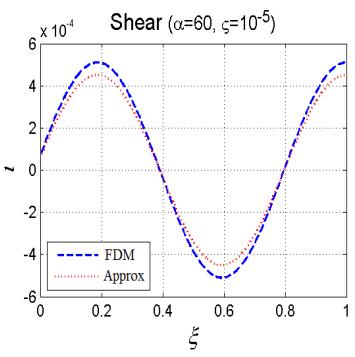

(o)

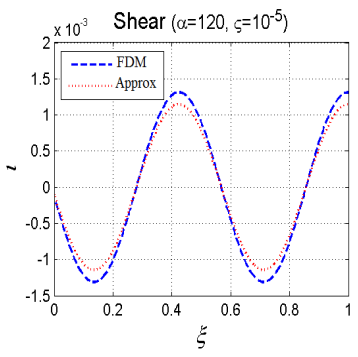

(s)

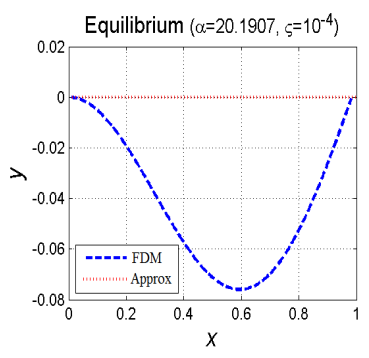

(d)

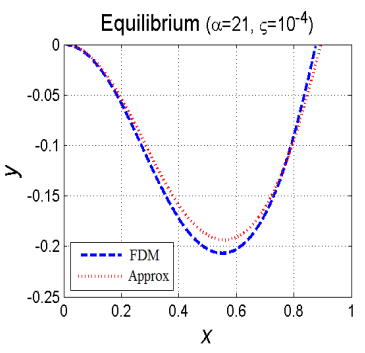

(h)

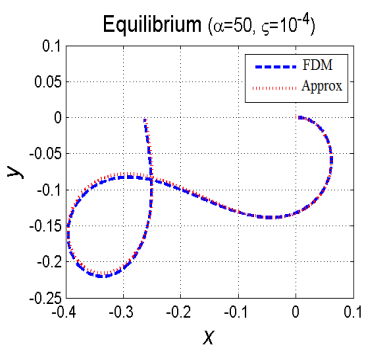

(1)

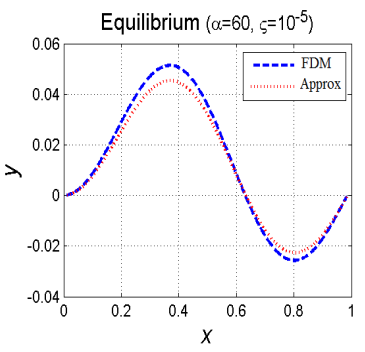

(p)

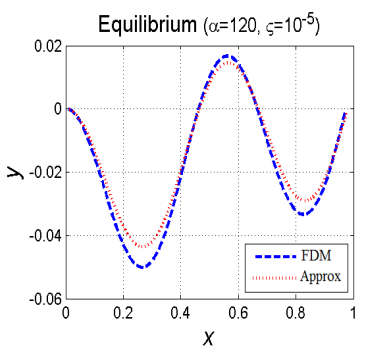

(t)

Fig. 15. Distributions of $\psi, \varepsilon$, and $\imath$ and equilibria of a fixed-pinned beam under an axial compressive force, when the dimensionless compressive force $\alpha$ is: (a) through (d), 20.1907; (e) through (h), 21; (i) through (l), 50; (m) through (p), 60; and (q) through (s), 120, calculated using the FDM (dashed) and the approximate method (dotted). 


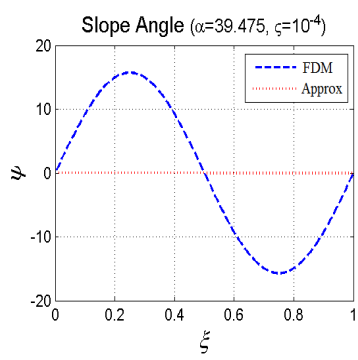

(a)

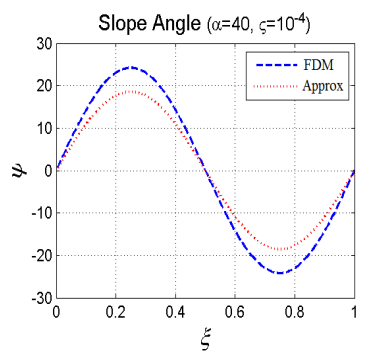

(e)

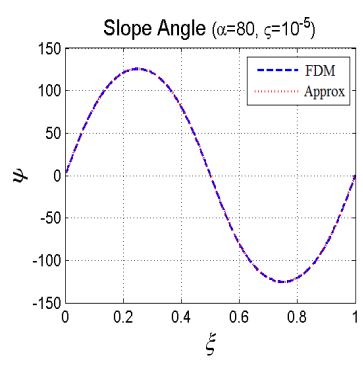

(i)

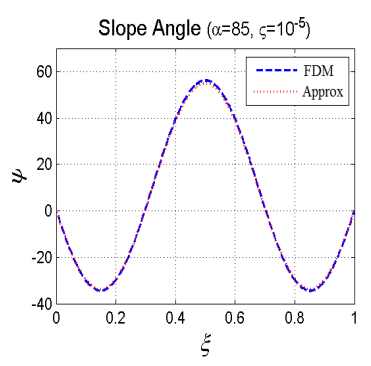

(m)

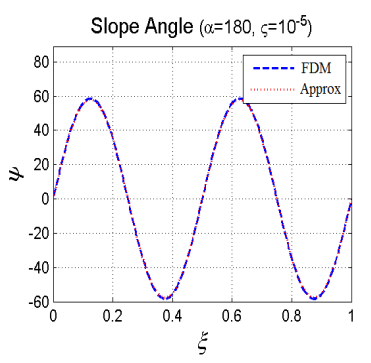

(q)

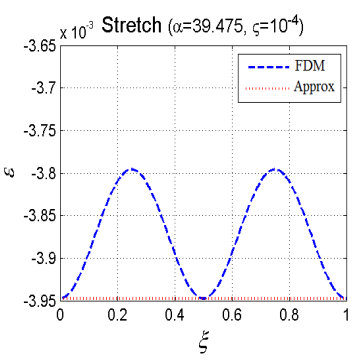

(b)

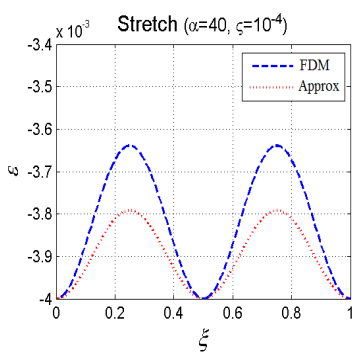

(f)

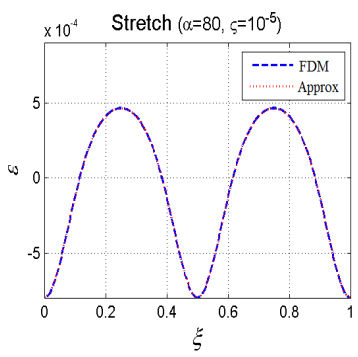

(j)

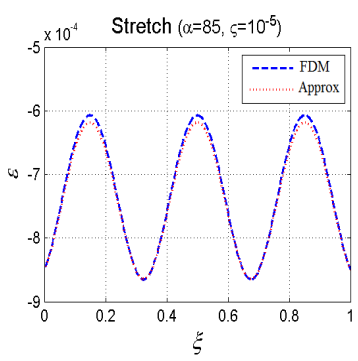

(n)

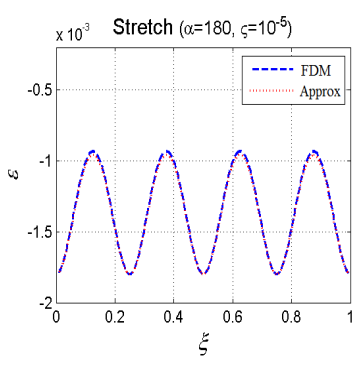

(r)

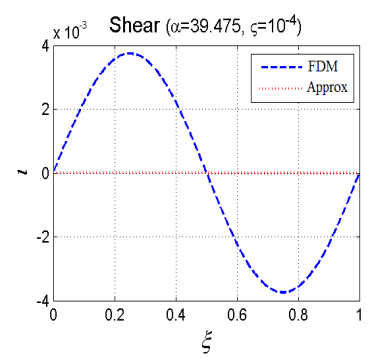

(c)

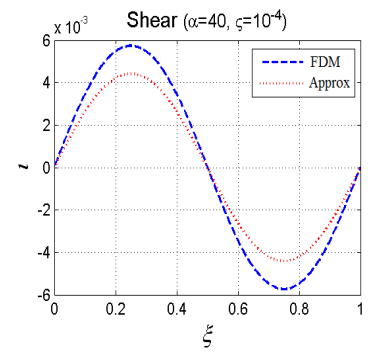

(g)

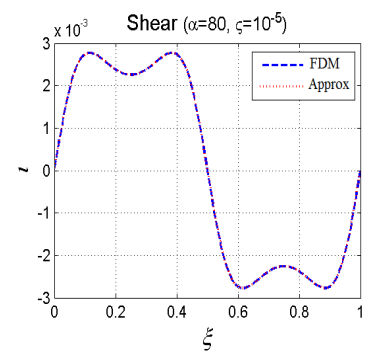

(k)

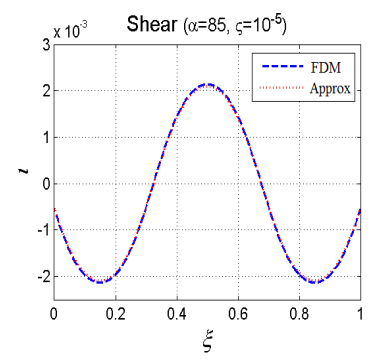

(o)

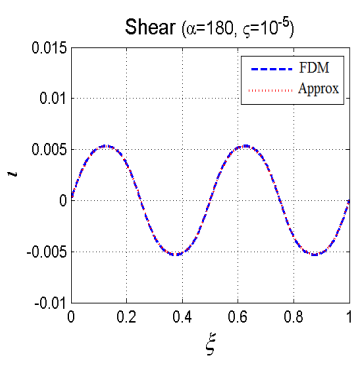

(s)

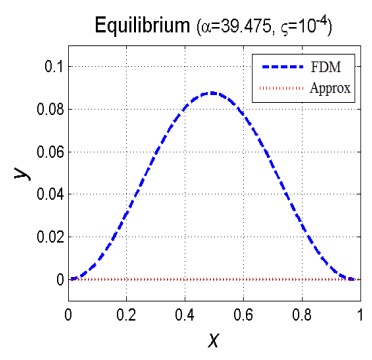

(d)

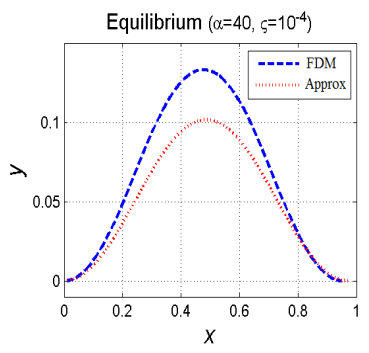

(h)

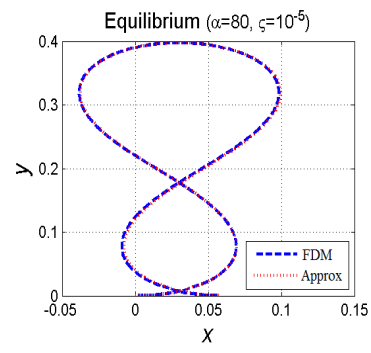

(1)

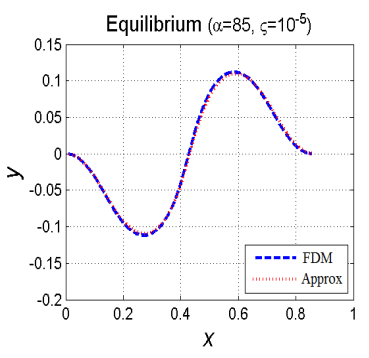

(p)

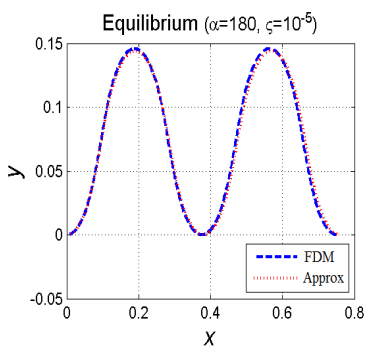

(t)

Fig. 16. Distributions of $\psi, \varepsilon$, and $\imath$ and equilibria of a fixed-fixed beam under an axial compressive force, when the dimensionless compressive force $\alpha$ is: (a) through (d), 39.475; (e) through (h), 40; (i) through (l), 80; (m) through (p), 85; and (q) through (s), 180, calculated using the FDM (dashed) and the approximate method (dotted). 
[7] H. Mobki, G. Rezazadeh, M. Sadeghi, F. Vakili-Tahami, and M.-M. Seyyed-Fakhrabadi, 2013, "A comprehensive study of stability in an electro-statically actuated micro-beam", International Journal of Non-Linear Mechanics, 48, pp. 78-85.

[8] Irvine, H. M., 1993, "Local Bending Stresses in Cables”, International Society of Offshore and Polar Engineers, 3, pp. 172-175.

[9] Zhu, W. D, Ren, H., and Xiao, C., 2011, “A Nonlinear Model of a Slack Cable with Bending Stiffness and Moving Ends with Application to Elevator Traveling and Compensation Cables", Journal of Applied Mechanics, 78, pp. 041017.

[10] Santillana, S., Virgin, L. N., and Plaut, R. H., 2005, "Equilibria and Vibration of a Heavy Pinched Loop", Journal of Sound and Vibration, 288, pp. 81-90.

[11] Svetlitsky, V. A., 2005, Statics of Rods, Springer.

[12] Kim, J. S., and Chirikjian, G. S., 2006, "Conformational Analysis of Stiff Chiral Polymers with End-constraints", Molecular Simulation, 32, December, pp. 1139-1154.

[13] Hodges, D. H., 2006, Nonlinear Composite Beam Theory, American Institute of Aeronautics and Astronautics.

[14] S.K. Kumar, R. Ganguli, and D. Harursampath, 2013, "Partial delamination modeling in composite beams using a finite element method", Finite Elements in Analysis and Design 76, pp. 1-12.

[15] I. Romero, M. Urrecha, and C. Cyron, 2014, "A torsion-free non-linear beam model", International Journal of NonLinear Mechanics 58, pp. 1-10.

[16] Simo, J. C., 1985, "A Finite Strain Beam Formulation, the three dimensional Problem. Part I", Computer methods in applied mechanics and engineering 49, pp. 55-70.

[17] Simo, J. C., and Vu-Quoc, L., 1986, “Three Dimensional Finite Strain Rod Model. Part II: Computational Aspects”, Computer methods in applied mechanics and engineering 58, pp. 79-116.

[18] Reissner, E., 1972, “On One-Dimensional Finite-Strain Beam Theory: the Plane Problem”, Journal of Applied Mathematics and Physics, 23, pp. 795-804.

[19] P.F. Pai, 2014, "Problems in geometrically exact modeling of highly flexible beams", Thin-walled structures, 76, pp. 6576.

[20] Crisfield, M. A., 1991, Non-linear Finite Element Analysis of Solids and Structures, Volume I \& II, John Wiley \& Sons, New York.

[21] Shabana, A. A., 1998, Dynamics of Multibody Systems, Cambridge University Press, Cambridge, pp. $270-344$.

[22] Von Dombrowski, S., 2002, "Analysis of Large Flexible Body Deformation in Multi-body Systems Using Absolute Coordinates", Multibody System Dynamics, 8, pp. 409-432.

[23] Timoshenko, S. P., and Gere, J. M., 1961, Theory of Elastic Stability, 2nd Edition, McGraw Hill, New York.

[24] Gere, J. M., and Timoshenko, S. P., 1997, Mechanics of Materials, PWS Pub Co., Boston, MA, pp. 461-465.

\section{Appendix A: Deformation of a Beam}

Consider the deformation of a beam segment shown in Fig. 17. The particle $a$ is located at the cross-section corresponding to the arc-length coordinate $s$, and the particle $b$ is located at that corresponding to the arc-length coordinate $s+d s$. Suppose that $a$ and $b$ have the cross-section coordinate $z$ from the centroid line. When the beam segment is undeformed, the length of $a b$ is $d s$, as shown in Fig. 17(a). After deformation, cross-sections of the beam segment may not be perpendicular to the centroid line any more. As shown in Fig. 17(b), the angle between the two normal planes of the centroid line at the two cross-sections is $d \theta$, and the radius of curvature of the centroid line is $\rho$. The stretch strain of the centroid line is $\varepsilon$, which is related to $\rho$ and $d \theta$ by

$$
\rho d \theta=(1+\varepsilon) d s
$$

Since the shear strain $\gamma$ is small, the length of $a b$ after deformation is $(\rho-z) d \theta+z(\gamma+d \gamma)-z \gamma=\rho d \theta-z d \theta+z d \gamma=$ $(1+\varepsilon) d s-z(d \theta-d \gamma)$, where Eq. (98) has been used. Hence, the strain along $a b$ is

$$
\varepsilon_{z}=\frac{(1+\varepsilon) d s-z(d \theta-d \gamma)-d s}{d s}=\varepsilon-z\left(\frac{d \theta}{d s}-\frac{d \gamma}{d s}\right)
$$

For linear elastic deformation, the axial stress distribution in the cross-section corresponding to the arc-length coordinate $s$ is

$$
\sigma_{z}=E \varepsilon_{z}=E \varepsilon-E z\left(\frac{d \theta}{d s}-\frac{d \gamma}{d s}\right)
$$




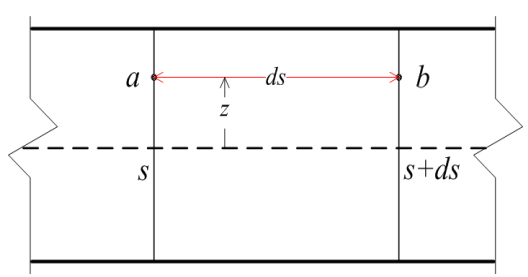

(a)

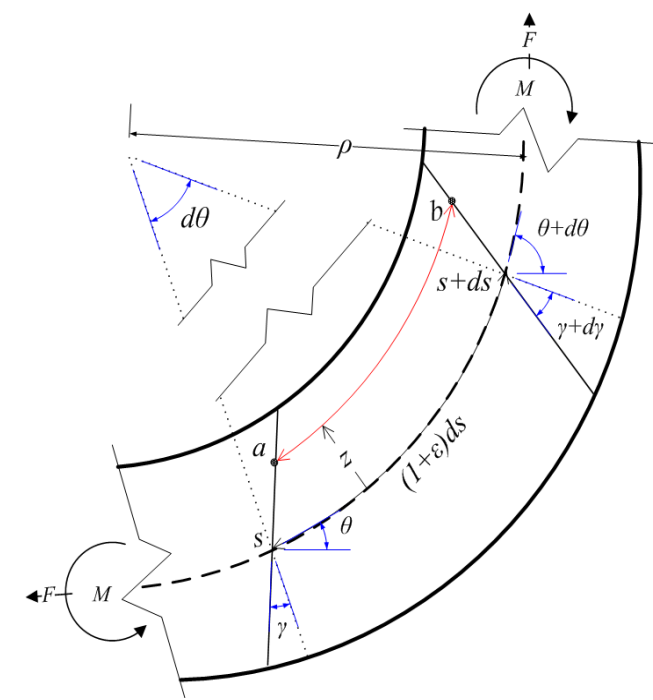

(b)

Fig. 17. Schematic of the deformation of a beam segment: (a) before deformation, and (b) after deformation.

Hence, the axial force on the cross-section is

$$
F=\int_{\mathcal{A}} \sigma_{z} d \mathrm{a}=E A \varepsilon
$$

where $\mathcal{A}$ indicates that the integration is performed on the cross-section, and the bending moment at the cross-section is

$$
M=\int_{\mathscr{A}} z \sigma_{z} d \mathrm{a}=-E I\left(\frac{d \theta}{d s}-\frac{d \gamma}{d s}\right)
$$

where

$$
\int_{\mathscr{A}} z d \mathrm{a}=0, \quad \int_{\mathfrak{A}} z^{2} d \mathrm{a}=I
$$

have been used. Hence, the elastic potential energy due to axial strains at the cross-section is $\frac{1}{2} \int_{\mathcal{A}} E \varepsilon_{z}^{2} d \mathrm{a}=\frac{1}{2} E A \varepsilon^{2}+$ $\frac{1}{2} E I\left(\frac{d \theta}{d s}-\frac{d \gamma}{d s}\right)^{2}$. When the shear strain $\gamma$ is uniform in the cross-section, as shown in Fig. 17(b), the elastic potential energy due to shear of the cross-section is $\frac{1}{2} \int_{\mathcal{A}} G \gamma^{2} d a=\frac{1}{2} G A \gamma^{2}$. Since the shear strain distribution is generally not uniform in the cross-section, the elastic potential energy due to shear of the cross-section is usually expressed as $\frac{1}{2} \frac{G A}{k} \gamma^{2}$ [24]. Hence, the elastic potential energy of the whole beam is

$$
\mathcal{P}=\frac{1}{2} \int_{0}^{L}\left\{E A(s) \varepsilon^{2}(s)+E I(s)\left[\frac{d \theta(s)}{d s}-\frac{d \gamma(s)}{d s}\right]^{2}+\frac{G A(s)}{k(s)} \gamma^{2}(s)\right\} d s
$$

